NASA Technical Memorandum 103273

ICOMP-90-22; AIAA-91-0413

\title{
Numerical Study of Shock-Wave/Boundary Layer Interactions in Premixed Hydrogen-Air Hypersonic Flows
}

Shaye Yungster Institute for Computational Mechanics in Propulsion Lewis Research Center

Cleveland, Ohio

Prepared for the 29th Aerospace Sciences Meeting sponsored by the American Institute of Aeronautics and Astronautics Reno, Nevada, January 7-10, 1991

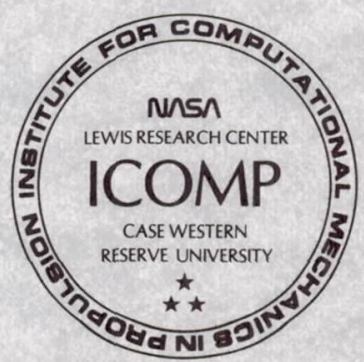




\title{
Numerical Study of Shock-Wave/Boundary Layer Interactions in Premixed Hydrogen-Air Hypersonic Flows
}

\author{
Shaye Yungster* \\ Institute for Computational Mechanics in Propulsion \\ NASA Lewis Research Center, Cleveland, OH 44135
}

\begin{abstract}
A computational study of shock wave/boundary layer interactions involving premixed combustible gases, and the resulting combustion processes is presented. The analysis is carried out using a new fully implicit, total variation diminishing (TVD) code developed for solving the fully coupled Reynolds-averaged Navier-Stokes equations and species continuity equations in an efficient manner. To accelerate the convergence of the basic iterative procedure, this code is combined with vector extrapolation methods. The chemical nonequilibrium processes are simulated by means of a finite-rate chemistry model for hydrogen-air combustion. Several validation test cases are presented and the results compared with experimental data or with other computational results. The code is then applied to study shock wave/boundary layer interactions in a ram accelerator configuration. Results indicate a new combustion mechanism in which a shock wave induces combustion in the boundary layer, which then propagates outwards and downstream. At higher Mach numbers, spontaneous ignition in part of the boundary layer is observed, which eventually extends along the entire boundary layer at still higher values of the Mach number.
\end{abstract}

\section{Introduction}

The interactions that occur when a shock wave impinges on a boundary layer have been extensively studied in the past. A summary of such research can be found for example in Refs. 1-3 for laminar and turbulent boundary layers. Most of these studies have concentrated on nonreacting airflows. In recent years, due to the current research in hypersonic airbreathing vehicles and hypervelocity mass launchers, interest has emerged in the study

*Senior Research Associate. Member AIA A

Copyright (C)1991 by the American Institute of Aeronautics and Astronautics, Inc. No copyright is asserted in the United States under Title 17, U.S. Code. The U.S. Government has a royaltyfree license to exercise all rights under the copyright claimed herein for Governmental purposes. All other rights are reserved by the copyright owner. of shock wave/boundary layer interactions involving combustible gas mixtures, and the resulting combustion processes. This type of interaction is present in many of the current concepts in hypersonic propulsion.

In a supersonic combustion ramjet (scramjet), shown schematically in Fig. 1a, a diffuser inlet decelerates the incoming air through a series of oblique shocks that increase its temperature and pressure. The fuel is injected in the combustor where it mixes with the hot air and combustion occurs at supersonic speeds. The combustion process in this concept is entirely dominated by the mixing rate, which is much slower than the reaction rates at typical combustor conditions. The fuel is injected nearly parallel to the air stream to reduce shock interaction losses. Parallel injection produces slow mixing, requiring long combustors. An early attempt to reduce combustor length led to a scramjet design in which some of the fuel was injected and premixed in the inlet upstream of the combustor ${ }^{4}$. This engine was unsuccessful, because separated flow between the combustor and the premix injection station allowed combustion to propagate upstream into the inlet ${ }^{5}$. This flow condition was probably caused by interactions between the shock wave system and the premixed combustible gases in the boundary layer.

The idea of premixing the fuel and air has also led to the concept of the oblique detonation wave engine (ODWE) shown schematically in Fig. 1b. In the ODWE, the fuel is injected into the air stream at a station well upstream of the combustor, where mixing occurs at a relatively low temperature ( except for the boundary layer where temperatures can be high). Ignition of the fuel/air mixture is achieved by means of a series of shock waves that increase its temperature until the ignition temperature is reached at some designed location. At this point, rapid chemical reactions release energy into the flowing stream. The energy addition will establish a detonation wave or a shock-deflagration system, depending primarily on the mixture composition and pressure. Since the combustion process is very fast in this case, the combustor can be very small and therefore significant savings in weight 
can be achieved. Early work on the ODWE concept was done by Townend ${ }^{6}$ and Morrison ${ }^{7}$, among others, and more recently by Menees et. al. ${ }^{8}$. The possibility of using shock-induced combustion has also been proposed as one of many alternative combustion modes for a new ramjetin-tube concept, developed at the University of Washington, known as the ram accelerator ${ }^{9-17}$. In this concept, a shaped projectile can, in principle, be accelerated efficiently to velocities up to $12 \mathrm{~km} / \mathrm{s}$ by means of detonation waves or other shock-induced combustion modes. An experimental ram accelerator device is currently operating at the University of Washington.

The gasdynamic principles that govern the flow and combustion processes in the ram accelerator (operating in the "oblique detonation mode") are similar to those of the ODWE concept, however the device is operated in a different manner.

In the oblique detonation ram accelerator operation mode (Fig. 1c), the centerbody is a projectile fired into a tube filled with a premixed gaseous fuel/oxidizer mixture. There is no propellant on board the projectile. Ignition of the fuel/oxidizer mixture is achieved by the same process described previously for the ODWE. Since the fuel and oxidizer in the ram accelerator concept are premixed, the difficulties in obtaining rapid and complete mixing encountered by the ODWE and the scramjet are circumvented. The combustion process creates a high pressure region over the back of the projectile, producing a thrust force. The pressure, composition, chemical energy density, and speed of sound of the mixture can be controlled to optimize the performance for a given flight condition. The ram accelerator concept has the potential for a number of applications, such as hypervelocity impact studies, direct launch to orbit of acceleration insensitive payloads, and hypersonic testing ${ }^{18-20}$.

It is clear that in all the above propulsion concepts, a boundary layer consisting of premixed combustible gases will exist at least in portions of the vehicle, and that the effects of a chemically reacting boundary layer and of shock wave/boundary layer interactions involving premixed combustible gases must be investigated. Previous theoretical and numerical studies of these propulsion concepts have not considered viscous effects. The objective of the present paper is, therefore, to analyze numerically such interactions. The results presented in this paper show that viscous interactions can be extremely important.

Perhaps the most powerful approach today to predicting shock wave/boundary layer interactions is to solve the Reynolds-averaged Navier-Stokes equations. Although computer codes presently in use are still evolving, numerical techniques have matured sufficiently to warrant their consideration in practical two-dimensional and threedimensional applications.
This paper presents a new CFD code that has been developed for solving the full Reynolds-averaged NavierStokes equations, including the reaction kinetics of a 7species, 8-step hydrogen/oxygen combustion model.

The code uses an iterative scheme that is based on the LU-SSOR implicit factorization scheme and a second order symmetric TVD scheme. The iterative scheme can further be combined with vector extrapolation methods to enhance its convergence properties. Extrapolation methods have been used in conjunction with iterative schemes in CFD codes, mostly for the Euler equations. As far as is known to the author, there have not been any applications in reacting flow problems so far. The extrapolation methods used in the present study are the Minimal Polynomial (MPE), and the Reduced Rank (RRE) Extrapolation.

The numerical formulation, iterative scheme, and extrapolation method used in the present work are described in the following sections.

\section{Numerical Formulation}

\section{Governing Equations}

The Reynolds-averaged Navier-Stokes equations for two-dimensional or axisymmetric flow are considered. For the case of chemically reacting flows, the global continuity equation is replaced by all the species continuity equations. They can be expressed in the following conservation form for a gas containing $n$ species and in general curvilinear coordinates $(\xi, \eta)$

$$
\frac{\partial \mathbf{Q}}{\partial t}+\frac{\partial\left(\mathbf{F}-\mathbf{F}_{v}\right)}{\partial \xi}+\frac{\partial\left(\mathbf{G}-\mathbf{G}_{v}\right)}{\partial \eta}+j\left(\mathbf{H}-\mathbf{H}_{v}\right)=\mathbf{W}
$$

where

$$
\mathbf{Q}=J^{-1}\left[\begin{array}{c}
\rho_{1} \\
\rho_{2} \\
\vdots \\
\rho_{n} \\
\rho u \\
\rho v \\
e
\end{array}\right], \quad \mathbf{F}=J^{-1}\left[\begin{array}{c}
\rho_{1} U \\
\rho_{2} U \\
\vdots \\
\rho_{n} U \\
\rho u U+\xi_{x} p \\
\rho v U+\xi_{y} p \\
U(e+p)
\end{array}\right]
$$

$$
\mathbf{G}=J^{-1}\left[\begin{array}{c}
\rho_{1} V \\
\rho_{2} V \\
\vdots \\
\rho_{n} V \\
\rho u V+\eta_{x} p \\
\rho v V+\eta_{y} p \\
V(e+p)
\end{array}\right], \quad \mathbf{H}=\frac{J^{-1}}{y}\left[\begin{array}{c}
\rho_{1} v \\
\rho_{2} v \\
\vdots \\
\rho_{n} v \\
\rho u v \\
\rho v^{2} \\
v(e+p)
\end{array}\right]
$$




$$
\mathbf{F}_{v}=\frac{J^{-1}}{R e}\left[\begin{array}{c}
-\rho_{1} U_{1}^{d} \\
-\rho_{2} U_{2}^{d} \\
\vdots \\
-\rho_{n} U_{n}^{d} \\
\xi_{x} \tau_{x x}+\xi_{y} \tau_{x y} \\
\xi_{x} \tau_{x y}+\xi_{y} \tau_{y y} \\
\xi_{x} \beta_{x}+\xi_{y} \beta_{y}
\end{array}\right], \quad \mathbf{H}_{v}=\frac{J^{-1}}{y R e}\left[\begin{array}{c}
-\rho_{1} v_{1}^{d} \\
-\rho_{2} v_{2}^{d} \\
\vdots \\
-\rho_{n} v_{n}^{d} \\
\tau_{x y} \\
\tau_{y y}-\tau_{\theta \theta} \\
\beta_{y}
\end{array}\right]
$$$$
\mathbf{G}_{v}=\frac{J^{-1}}{R e}\left[\begin{array}{c}
-\rho_{1} V_{1}^{d} \\
-\rho_{2} V_{2}^{d} \\
\vdots \\
-\rho_{n} V_{n}^{d} \\
\eta_{x} \tau_{x x}+\eta_{y} \tau_{x y} \\
\eta_{x} \tau_{x y}+\eta_{y} \tau_{y y} \\
\eta_{x} \beta_{x}+\eta_{y} \beta_{y}
\end{array}\right], \quad \mathbf{W}=J^{-1}\left[\begin{array}{c}
w_{1} \\
w_{2} \\
\vdots \\
w_{n} \\
0 \\
0 \\
0
\end{array}\right]
$$

and

$$
\begin{aligned}
& \beta_{x}=u \tau_{x x}+v \tau_{x y}-q_{x} \\
& \beta_{y}=u \tau_{x y}+v \tau_{y y}-q_{y}
\end{aligned}
$$

The stress tensor components are given by

$$
\begin{gathered}
\tau_{x x}=\mu\left[\frac{4}{3}\left(\xi_{x} u_{\xi}+\eta_{x} u_{\eta}\right)-\frac{2}{3}\left(\xi_{y} v_{\xi}+\eta_{y} v_{\eta}\right)-\frac{2}{3} \frac{v}{y}\right] \\
\tau_{y y}=\mu\left[\frac{4}{3}\left(\xi_{y} v_{\xi}+\eta_{y} v_{\eta}\right)-\frac{2}{3}\left(\xi_{x} u_{\xi}+\eta_{x} u_{\eta}\right)-\frac{2}{3} \frac{v}{y}\right] \\
\tau_{\theta \theta}=\mu\left[-\frac{2}{3}\left(\xi_{x} u_{\xi}+\eta_{x} u_{\eta}+\xi_{y} v_{\xi}+\eta_{y} v_{\eta}\right)+\frac{4}{3} \frac{v}{y}\right] \\
\tau_{x y}=\mu\left[\xi_{y} u_{\xi}+\eta_{y} u_{\eta}+\xi_{x} v_{\xi}+\eta_{x} u_{\eta}\right]
\end{gathered}
$$

where $M_{i}$ is the molecular weight of the $i$ th species, and $R$ is the universal gas constant. The temperature $T$, is determined from the definition of the total energy :

$$
\sum_{i=1}^{n} c_{i} \int^{T} c_{v_{i}} d T=\frac{e}{\rho}-\frac{1}{2}\left(u^{2}+v^{2}\right)-\sum_{i=1}^{n} c_{i} h_{i}^{0}
$$

where $c_{v_{i}}$ is the specific heat at constant volume of the $i$ th species, and $h_{i}^{0}$ is the heat of formation for species $i$.

\section{Thermodynamics and Transport Properties}

$$
\begin{aligned}
& q_{x}=-k \frac{\partial T}{\partial x}+\sum_{i=1}^{n} \rho_{i} u_{i}^{d} h_{i} \\
& q_{y}=-k \frac{\partial T}{\partial y}+\sum_{i=1}^{n} \rho_{i} v_{i}^{d} h_{i}
\end{aligned}
$$

The equations describe two-dimensional flow if $j=0$ and axisymmetric flow if $j=1$. The variables are the velocity components $u$ and $v$, the pressure $p$, the energy per unit volume $e$, and the density of the $i$ th species $\rho_{i}$, with $\rho=\sum_{i=1}^{n} \rho_{i}$. The terms $w_{i}$ represent the production of species from chemical reactions and are calculated by standard methods. The variable $y$ is the cylindrical radius. The grid Jacobian J and the contravariant velocities $U$ and $V$ are defined as follows

$$
J^{-1}=x_{\xi} y_{\eta}-x_{\eta} y_{\xi}
$$

Expressions for the specific heat as a function of temperature are obtained from the following polynomial fit

$$
\frac{c_{p_{i}}}{R}=A_{1}+A_{2} T+A_{3} T^{2}+A_{4} T^{3}+A_{5} T^{4}
$$

where $c_{p_{i}}$ is the specific heat at constant pressure of the $i$ th species, and $A_{1}, \ldots, A_{5}$ are constants. The thermal conductivity and viscosity for each species are determined by similar fourth-order polynomials of temperature. The coefficients of these polynomials are supplied by McBride and Shuen ${ }^{21}$ and are valid up to a temperature of $6000^{\circ} \mathrm{K}$. The thermal conductivity and viscosity of the mixture are calculated using Wilke's mixing rule ${ }^{22}$.

The binary mass diffusivity $D_{i j}$ between species $\mathrm{i}$ and $\mathrm{j}$ is obtained using the Chapman-Enskog theory in conjunction with the Lennard-Jones intermolecular potential functions ${ }^{22}$. 


\section{Combustion and Turbulence Model}

In the present study, a 7 -species, 8-step reaction mechanism for hydrogen-oxygen combustion is adopted. This model is a reduced reaction mechanism obtained from more complete models by the exclusion of the reactions involving $\mathrm{H}_{2} \mathrm{O}_{2}$ and $\mathrm{HO}_{2}$, which could be important in low temperature ignition studies. A complete description of the reduced model can be found in Refs. 13 and 23, together with a discussion of its accuracy and range of application. Further evidence supporting the validity of this combustion model has recently been presented by Sekar et. al. ${ }^{24}$ In their study of chemically reacting mixing layers, they compared the performance of various combustion models. In particular they found that the results obtained with a 7-species, 7-step reaction mechanism, very similar to the one used in the present study, were nearly identical to those obtained with a more complete 9 -species, 18-step model at various inflow conditions.

The turbulent model adopted in the present study is the Baldwin-Lomax algebraic eddy viscosity model ${ }^{25}$ and assumes constant turbulent Prandtl and Schmidt numbers $\left(P r_{t}=S c_{t}=0.9\right)$. This model is chosen for its simplicity and computational efficiency.

The interactions between turbulence and chemistry, which enter into the numerical formulation through the source term $w_{i}$, represent a very difficult problem. To account for such interaction effects would require a closure method such as the probability density function (PDF) approach or a direct numerical simulation (DNS). Since effective PDF closure methods are not yet available and DNS methods are currently applicable only to relatively simple flows, the interactions between turbulence and chemistry are not considered in the present study.

\section{Numerical Method}

The equation set describing chemically reacting flows is difficult to solve because it is mathematically stiff. There are currently two approaches to solving chemically reacting flows. One approach is to uncouple the fluid dynamics equations from the rate equations. Each timestep consists of a fluid dynamics step with frozen chemistry followed by a chemical reaction step (or several small steps) without flow interaction ${ }^{26}$. The uncoupled method has the advantage of of being more flexible since it can employ different algorithms for different physical processes. However, since in most chemically reacting flows the coupling between chemistry and flow is strong, problems with achieving convergence have been encountered. The second approach solves the fully coupled equation set simultaneously. This approach has the advantage of directly taking into account the close coupling between flow and chemistry.
In this paper the fully coupled equation set is solved using a new fully implicit total variation diminishing (TVD) code. The new iterative scheme is a modification of the author's previous predictor corrector explicit code $\mathrm{c}^{13,23}$. As mentioned in the introduction, the iterative scheme can also be combined with the RRE or MPE vector extrapolation techniques to enhance its convergence. A description of the iterative scheme and of one of the extrapolation methods, namely RRE, is given below.

\section{LU-SSOR Scheme}

An unfactored linearized implicit scheme for equation (1) can be written as

$$
\left[\mathbf{I}+\beta \Delta t\left(D_{\xi} \mathbf{A}+D_{\eta} \mathbf{B}-\mathbf{C}\right)\right] \Delta \mathbf{Q}=-\Delta t[\mathbf{R H S}]
$$

where RHS is the right hand side residual

$$
\mathbf{R H S}=D_{\xi}\left(\mathbf{F}-\mathbf{F}_{v}\right)+D_{\eta}\left(\mathbf{G}-\mathbf{G}_{v}\right)+j\left(\mathbf{H}-\mathbf{H}_{v}\right)-\mathbf{W}
$$

In the above, $D_{\xi}$ and $D_{\eta}$ are difference operators that approximate $\frac{\partial}{\partial x}$ and $\frac{\partial}{\partial y}$, and $\Delta \mathbf{Q}$ is the correction

$$
\Delta \mathrm{Q}=\mathrm{Q}^{n+1}-\mathrm{Q}^{n}
$$

The terms A, B, C are the following Jacobian matrices:

$$
\mathbf{A}=\frac{\partial \mathbf{F}}{\partial \mathbf{Q}}, \quad \mathbf{B}=\frac{\partial \mathbf{G}}{\partial \mathbf{Q}}, \quad \mathbf{C}=\frac{\partial \mathbf{W}}{\partial \mathbf{Q}},
$$

The unfactored implicit scheme in Eq. 12 produces a large block banded matrix that is very costly to invert and forces recourse to either an approximate factorization method or an iterative solution method. The most efficient approximate factorization involves a lower-upper (LU) decomposition ${ }^{27}$. A variation of the LU decomposition method, known as the LU-SSOR scheme, was developed by Yoon and Jameson for nonreacting flows ${ }^{28,29}$ and later extended to reacting flows by Shuen and Yoon ${ }^{30}$. In the present paper the LU-SSOR scheme is adopted to solve Eq. 1. The LU-SSOR implicit factorization scheme can be written as

$$
\mathrm{LT}^{-1} \mathrm{U} \Delta \mathrm{Q}=-\Delta t[\mathrm{RHS}]
$$

where $\mathbf{L}$ and $\mathbf{U}$ are the lower and upper operators

$$
\begin{gathered}
\mathbf{L}=\mathbf{I}+\beta \Delta t\left[D_{\xi}^{-} \mathbf{A}^{+}+D_{\eta}^{-} \mathbf{B}^{+}-\mathbf{A}^{-}-\mathbf{B}^{-}-\mathbf{C}\right] \\
\mathbf{U}=\mathbf{I}+\beta \Delta t\left[D_{\xi}^{+} \mathbf{A}^{-}+D_{\eta}^{+} \mathbf{B}^{-}+\mathbf{A}^{+}+\mathbf{B}^{+}\right] \\
\mathbf{T}=\mathbf{I}+\beta \Delta t\left[\mathbf{A}^{+}+\mathbf{B}^{+}-\mathbf{A}^{-}-\mathbf{B}^{-}\right]
\end{gathered}
$$

Here, $D_{\xi}^{-}$and $D_{\eta}^{-}$are the backward difference operators, and $D_{\xi}^{+}$and $D_{\eta}^{+}$are the forward difference operators. Two-point operators are used in the present work. The Jacobian matrices are aproximately constructed so that 
the eigenvalues of $(+)$ matrices are nonnegative and those of (-) matrices are nonpositive. The most commonly used expressions are

$$
\mathbf{A}^{ \pm}=\frac{1}{2}[\mathbf{A} \pm \Lambda(\mathbf{A}) \mathbf{I}]
$$

and

$$
\Lambda(\mathbf{A})=\kappa[\max (|\lambda(\mathbf{A})|)], \quad \kappa \geq 1
$$

where $\lambda(\mathbf{A})$ represent the eigenvalues of the Jacobian matrix A.

In the present work, the right-hand-side residual RHS in Eq. 16 is calculated using the modified flux approach of Yee and Harten for obtaining a second-order symmetric TVD scheme ${ }^{31,32}$. This represents a departure from previous work in which the residual is evaluated using central difference operators and a fourth-order artificial dissipation model as in Shuen and Yoon ${ }^{30}$, or a flux-limited dissipation model as in Park and Yoon ${ }^{33}$. In a recent paper, Tsai and Hsieh ${ }^{34}$ used the Van Leer's flux vector splitting technique for discretizing the residual. Clearly, a characteristic-based scheme is to be preferred in order to achieve the best possible resolution of discontinuities and good convergence rates.

\section{Second-order Symmetric TVD Scheme}

Following Yee and Harten's modified flux approach, the derivatives of the residual RHS in Eq. 16 are evaluated as follows

$$
\begin{aligned}
D_{\xi} \mathbf{F} & =\frac{\tilde{\mathbf{F}}_{j+\frac{1}{2}, k}-\tilde{\mathbf{F}}_{j-\frac{1}{2}, k}}{\Delta \xi} \\
D_{\eta} \mathbf{G} & =\frac{\tilde{\mathbf{G}}_{j, k+\frac{1}{2}}-\tilde{\mathbf{G}}_{j, k-\frac{1}{2}}}{\Delta \eta}
\end{aligned}
$$

The functions $\tilde{\mathbf{F}}_{j+\frac{1}{2}, k}$ and $\tilde{\mathbf{G}}_{j, k+\frac{1}{2}}$ are the numerical fluxes in the $\xi$ and $\eta$ directions evaluated at $\left(j+\frac{1}{2}, k\right)$ and $\left(j, k+\frac{1}{2}\right)$, respectively. Typically, $\tilde{\mathbf{F}}_{j+\frac{1}{2}, k}$ can be expressed as

$$
\tilde{\mathbf{F}}_{j+\frac{1}{2}, k}=\frac{1}{2}\left(\tilde{\mathbf{F}}_{j, k}+\tilde{\mathbf{F}}_{j+1, k}+\mathbf{R}_{j+\frac{1}{2}} \boldsymbol{\Phi}_{j+\frac{1}{2}}\right)
$$

Here $\mathbf{R}_{j+\frac{1}{2}}$ denotes the matrix of eigenvectors of the flux Jacobian matrix $\mathbf{A}$ evaluated at some symmetric average of $\mathbf{Q}_{j, k}$ and $\mathbf{Q}_{j+1, k}$, denoted as $\mathbf{Q}_{j+\frac{1}{2}}$. Similarly, one can define the numerical flux $\tilde{\mathrm{G}}_{j, k+\frac{1}{2}}$ in this manner. The viscous terms are evaluated using central differences.

The elements, $\phi_{j+\frac{1}{2}}^{l}$, of the dissipation vector $\Phi_{j+\frac{1}{2}}$ are:

$$
\begin{gathered}
\phi_{j+\frac{1}{2}}^{l}=-\Psi\left(a_{j+\frac{1}{2}}^{l}\right)\left[\alpha_{j+\frac{1}{2}}^{l}-\hat{Q}_{j+\frac{1}{2}}^{l}\right] \\
\alpha_{j+\frac{1}{2}}=\mathbf{R}_{j+\frac{1}{2}}^{-1}\left(\mathbf{Q}_{j+1, k}-\mathbf{Q}_{j, k}\right)
\end{gathered}
$$

Here $a_{j+\frac{1}{2}}^{l}$ denotes the eigenvalues of $\mathbf{A}$ evaluated at $\mathbf{Q}_{j+\frac{1}{2}}$, and $\alpha_{j+\frac{1}{2}}^{l}$ denotes the elements of the vector $\boldsymbol{\alpha}_{j+\frac{1}{2}}$. The function $\Psi$ is :

$$
\Psi(z)= \begin{cases}|z| & |z| \geq \epsilon \\ \frac{\left(z^{2}+\epsilon^{2}\right)}{2 \epsilon} & |z|<\epsilon\end{cases}
$$

The term $\epsilon$ in Eq. 26 is taken to be a function of the velocity and sound speed ${ }^{35}$

$$
\begin{aligned}
\epsilon_{j+\frac{1}{2}}=\tilde{\epsilon}\left[\left|U_{j+\frac{1}{2}}\right|\right. & +\left|V_{j+\frac{1}{2}}\right| \\
& \left.+\frac{1}{2}\left(\left(\sqrt{\xi_{x}^{2}+\xi_{y}^{2}}+\sqrt{\eta_{x}^{2}+\eta_{y}^{2}}\right) a\right)_{j+\frac{1}{2}}\right]
\end{aligned}
$$

where $a$ is the frozen sound speed, and $\tilde{\epsilon}$ is a small number in the range $0 \leq \tilde{\epsilon} \leq 0.4$, which controls the convergence rate and the sharpness of discontinuities. The smaller the value of $\tilde{\epsilon}$ is, the slower the convergence rate and the smaller the numerical dissipation being added. The "limiter" functions $\hat{Q}_{j+\frac{1}{2}}$ used in this study are the "minmod" limiter given by

$$
\hat{Q}_{j+\frac{1}{2}}^{l}=\operatorname{minmod}\left(\alpha_{j-\frac{1}{2}}^{l}, \alpha_{j+\frac{1}{2}}^{l}, \alpha_{j+\frac{3}{2}}^{l}\right)
$$

and a more compressive limiter, known as "superbee," given by

$$
\begin{array}{r}
\hat{Q}_{j+\frac{1}{2}}^{l}=\operatorname{minmod}\left[2 \alpha_{j-\frac{1}{2}}^{l}, 2 \alpha_{j+\frac{1}{2}}^{l}, 2 \alpha_{j+\frac{3}{2}}^{l}\right. \\
\left., \frac{1}{2}\left(\alpha_{j-\frac{1}{2}}^{l}+\alpha_{j+\frac{3}{2}}^{l}\right)\right]
\end{array}
$$

The minmod function of a list of arguments is equal to the smallest number in absolute value if the list of arguments is of the same sign, or is equal to zero if any arguments are of opposite sign. Alternative forms of the "limiter" function are given in Ref. 35. Except where indicated, all the results presented here were obtained with the minmod limiter given by Eq. 28 .

The eigenvalues and eigenvectors of the fully coupled chemically reacting equations in generalized coordinates were obtained in a previous work ${ }^{13,15}$ and used for calculating the vectors $\mathbf{R} \Phi$ appearing in Eq. 23. The resulting expressions for $\mathbf{R} \Phi$ are given in Refs. 13 and 15. This scheme is second-order accurate in space and is suitable for steady-state calculations.

\section{Extrapolation Method for Convergence Acceleration}

There are several extrapolation methods in the literature for achieving faster convergence rates. Of these methods, MPE and RRE seem to be the most efficient as far as the amount of computing and storage requirements are concerned. In the present study, both MPE and RRE are used in the so called "cycling" mode through their 
implementations given in the recent work of Sidi ${ }^{36,37}$. A brief description of the cycling mode is given below. More details and further references concerning these methods and others are given in Refs. 36,37.

Step 1. Given an initial approximation $\mathbf{Q}^{0}$, use the iterative scheme to generate the sequence of approximations $\mathrm{Q}^{1}, \mathrm{Q}^{2}, \ldots, \mathrm{Q}^{N_{0}}$, and set $\mathrm{Q}^{0} \leftarrow \mathrm{Q}^{N_{0}}$, and $q=1$. Here, $N_{0}$ is a given positive integer and $q$ is the number of cycles.

Step 2. Beginning now with $\mathrm{Q}^{0}$, generate the sequence $\mathrm{Q}^{1}, \mathrm{Q}^{2}, \ldots, \mathrm{Q}^{K M A X+1}$, for some fixed integer KMAX.

Step 3. Apply RRE (or MPE) to this sequence to obtain the approximation $\mathrm{S}^{q+1} \equiv \mathrm{S}_{0, K M A X}$. (The determination of $\mathrm{S}_{0, K M A X}$ from $\mathrm{Q}^{1}, \mathrm{Q}^{2}, \ldots, \mathrm{Q}^{K M A X+1}$ will be described later).

Step 4. If $\mathbf{S}^{q+1}$ is a satisfactory approximation, stop; otherwise replace $\mathrm{Q}^{0}$ by $\mathrm{S}^{q+1}$, and $q$ by $q+1$, and go to step 2 .

In general, it is observed that the sequence of approximations $\mathbf{S}^{1}, \mathbf{S}^{2}, \ldots$, has better convergence properties than the sequence obtained from the iterative scheme alone. (For linearly generated vector sequences, it can be shown in some cases that the logarithm of the norm of the residual associated with $\mathbf{S}^{q}$ decreases linearly as a function of $q$. For nonlinearly generated sequences, however, no rigorous error analysis exists so far).

A brief outline of RRE is given below, for MPE see Ref. 36,37 .

Given the vector sequence $\mathrm{Q}^{0}, \mathrm{Q}^{1}, \ldots, \mathrm{Q}^{k+1}$, with $k=$ $K M A X$, compute the differences

$$
\Delta \mathrm{Q}^{j}=\mathrm{Q}^{j+1}-\mathrm{Q}^{j}, \quad j=0,1, \ldots, k .
$$

Next, determine the scalars $\gamma_{0}, \gamma_{1}, \ldots, \gamma_{k}$ by solving the constrained least-squares problem

$$
\operatorname{minimize}\left\|\sum_{j=0}^{k} \gamma_{j} \Delta \mathrm{Q}^{j}\right\|
$$

subject to $\sum_{j=0}^{k} \gamma_{j}=1$.

Finally, set

$$
\mathrm{S}_{0, k}=\sum_{j=0}^{k} \gamma_{j} \mathbf{Q}^{j}
$$

Although the original definition of RRE is different, the definition given above is equivalent to it and results in an implementation that is more stable numerically.

\section{Results}

The numerical scheme described above, has been validated by using benchmark test cases for which experimental or numerical results are available. Several such validation test cases will be presented here, preceding the discussion of shock wave/boundary layer interactions in premixed $\mathrm{H}_{2}$-air hypersonic flows. One of the test cases was treated also by combining the basic iterative scheme with RRE and/or MPE, and the results for this case will be presented at the end of this section.

\section{Benchmark test cases: Nonreacting flows}

The first benchmark test case consisted of a $M=5$ nonreacting flow past a two-dimensional wedge configuration. The results are compared with those obtained using the "RPLUS" code developed by Shuen and Yoon ${ }^{30}$, which is fully implicit and employs a centered differenced scheme with artificial dissipation. Figure 2 shows a comparison between the pressure contours obtained by the two methods, and Fig. 3 shows the pressure distribution along the wedge surface. Excellent agreement between the two results is obtained, however, the present method captures a crisper nose shock. Figure 4 shows the variation in the skin friction coefficient along the body surface. The main differences in the results occur near the tip of the nose, where the grid resolution of the boundary layer is poor, and in the shock wave/boundary layer interaction region. Note that the present method predicts a larger drop in the skin friction coefficient at the interaction, and slightly negative values of the skin friction coefficient at a couple of gridpoints. This difference is due to excessive artificial dissipation introduced by the differencing scheme used in RPLUS.

The next benchmark test cases that were considered involve two-dimensional shock wave/boundary layer interactions in laminar and turbulent nonreacting airflows. For the laminar interaction, a flat plate and a $3^{\circ}$ shock generator configuration in a $M=4$ airflow is considered. The Reynolds number per unit length and the free-stream static pressure are $9.54 \times 10^{6} / \mathrm{m}$ and $206.82 \mathrm{kPa}$, respectively. Under these conditions, which reproduce those of the experiments conducted by Skebe et. al. ${ }^{38}$, a laminar boundary layer exists between the leading edge of the flat plate and the interaction region. Figure 5 shows pressure contours for the laminar shock wave/boundary layer interaction. This calculation was done using the "superbee" limiter on a $138 \times 75$ grid. The solution clearly shows the incident-, separation-, and reattachment-shock, as well as the boundary layer leading edge shock. The surface static pressure, and skin friction coefficient plotted in Fig. 6, are compared with the experimental results of Skebe et. al. ${ }^{36}$ Note that the calculations predict a smaller separation bubble length relative to the experimental one. Also, beyond $x=11 \mathrm{~cm}$, the boundary layer becomes transitional and the experimental skin friction data start to depart from the laminar calculation.

For the turbulent interaction, a flat plate and a $13^{\circ}$ shock generator configuration in a $M=3$ airflow is considered. The Reynolds number based on the boundary layer thickness $\left(\delta_{0}\right)$ ahead of the interaction is about $10^{6}$. 
Experimental data for this configuration was obtained by Reda and Murphy ${ }^{39}$. A $103 \times 87$ grid was used in this calculation. Figure 7 shows the skin friction coefficient and the ratio of surface pressure to free-stream total pressure plotted versus distance along the surface (nondimensionalized by $\delta_{0}$ ). Here, $x_{0}$ is taken as the theoretical inviscid flow impingement point for the incident shockwave. Note that the location of separation is well predicted, however, the computed location of reattachment was approximately one boundary layer thickness downstream relative to the experimental measurements. The calculated pressure variation is in close agreement with the experimental data.

\section{Benchmark test cases: Reacting flows}

In previous publications, the author presented a series of inviscid test cases conducted on the exothermic blunt body flow problem ${ }^{13,15}$. This type of flow, which consists of blunt projectiles flying into detonable gas mixtures, was experimentally investigated in the mid 1960's and early 1970 's. These experiments are extremely useful for testing CFD codes in a wide range of shock-induced combustion phenomena; from decoupled shock-deflagration systems, to overdriven detonation waves. The computations presented in Refs. 13,15 on the exothermic blunt body flow have been successfully repeated using the present numerical method (assuming inviscid flow). The results obtained are identical to those previously reported, and therefore, they will not be repeated here.

The final test problem considered in the present paper is the combustion of a premixed stoichiometric hydrogenair supersonic flow over a compression corner. Figure 8 shows pressure contours for inflow conditions of $T_{\infty}=$ $900 K$ and $M=4.5$. The leading-edge shock and the ramp shock-deflagration wave are clearly seen. The combustion process behind the ramp shock causes it to bend upward. The reason for this rotation of the wave has been pointed out by Cambier ${ }^{25}$ and is explained as follows. The heat from combustion accelerates the flow in a direction normal to the shock wave. Since the flow deflection remains the same for a fixed ramp angle, and since the tangential component of the velocity remains the same across the discontinuity, there must be an increase of the wave angle towards a normal wave.

Figure 9 shows the variation of pressure and temperature along the gridline located $0.2 \mathrm{~cm}$ from the base of the ramp. The results are compared with those obtained by Shuen and Yoon using an 8-species, 14-step combustion model $^{30}$. Figure 10 shows a comparison of species mass fraction distribution along the same gridline. The small differences observed in Figs. 9 and 10 can be attributed mainly to the different combustion models and differencing schemes used in the two studies. Figure 11 shows the convergence histories for the two methods. Note that the present TVD scheme converges faster than the centered differenced RPLUS code. This improvement in convergence is typical of characteristic-based schemes, and new fully upwinded versions of RPLUS show similar improvements in convergence rate over the centered differenced version $^{34}$.

\section{Shock-wave/boundary layer interactions in premixed $\mathrm{H}_{2}$-air hypersonic flow}

The interactions between a shock-wave and a boundary layer in premixed $\mathrm{H}_{2}$-air hypersonic flows are investigated for a ram accelerator configuration having dimensions similar to those of the experimental device presently operating at the University of Washington ${ }^{9-17}$. Only the frontal part of the projectile $(0 \leq x / L \leq 0.61)$ is considered in the present study. Here, $L$ is the length of a complete projectile and is set to a value of $L=15 \mathrm{~cm}$. The geometry and inflow conditions are shown in Fig. 12. A constant projectile surface temperature $T_{w}=600^{\circ} \mathrm{K}$ is assumed in all of the calculations. Also, the flow is assumed to be fully turbulent along the entire projectile.

Computations were conducted for a projectile moving at $M=6.7$ and a fill pressure of 1 atm, for which it was assumed first that no chemical reactions occur (the chemistry part was switched "off"). Figure 13 shows temperature contours obtained for the above conditions with a $127 \times 45$ grid. For clarity, the plot is magnified in the vertical direction by a factor of 2 . Note the high temperatures that are created in the boundary layer immediately behind the shock-wave/boundary layer interaction region. When the chemical reactions are switched "on", combustion will start in this region, as shown in Figs. 14 and 15. Figure 14 shows temperature contours after 100 iterations, starting from the nonreacting solution. The combustion process starts at the boundary layer and propagates outwards and downstream. Figure 15 shows the converged solution. A stable shock-deflagration system is established in this case. At this point, it is interesting to compare the resulting skin friction coefficient for reacting and nonreacting flow. This comparison is presented in Fig. 16. In the nonreacting flow case, the skin friction coefficient increases across the interaction region. This increase is typical of high Mach number flows and can be explained as follows. The shear stress, $\tau$, in the wall layer is given by

$$
-\rho \overline{u^{\prime} v^{\prime}}+\frac{\mu}{R e} \frac{\partial u}{\partial n}=\tau
$$

Assuming that the pressure gradient and inertia terms give higher-order corrections even in the interaction region, then it can be shown ${ }^{40}$ that the shear stress is constant across the wall layer (constant stress layer), and therefore

$$
\tau_{w}=\tau=-\rho \overline{u^{\prime} v^{\prime}}+\frac{\mu}{R e} \frac{\partial u}{\partial n}
$$


where $\tau_{w}$ is the wall shear stress. Consider then a streamline somewhat outside the viscous sublayer where the viscous stress becomes small compared to the Reynolds stress. Along this streamline, as the flow is decelerated across the shock, $\overline{u^{\prime} v^{\prime}}$ decreases, but the density, $\rho$, increases. At high Mach numbers, the jump in density across the shock wave is large and the Reynolds stress increases.

When combustion takes place behind the interaction, the density increases first across the shock but decreases immediately behind it due to the combustion process, which produces a high temperature-low density boundary layer. The result is a "spike" shown in Fig. 16, and a reduction in the skin friction coefficient downstream. Also, due to the reduced turbulent stress intensity behind the combustion front, the boundary layer separates when the second reflected shock wave impinges on the projectile surface.

Computational results from a grid refinement study are shown in Fig. 17, which shows the skin friction coefficient in the shock wave/boundary layer interaction region for the baseline grid $(127 \times 45)$, and for coarser $(101 \times 37)$, and finer $(170 \times 59)$ grids. The $(101 \times 37)$ grid is too coarse to adequately resolve the interaction process. As shown in Fig. 17, details of the interaction region are generally resolved with the baseline grid used in this study, although a small difference exists between the baseline and finer grids at the interaction and behind the combustion front. All the subsequent calculations (described below) were obtained with the $127 \times 45$ grid.

For a higher Mach number flow $(M=7.2)$, combustion begins prematurely in the boundary layer at the nose region of the projectile, as shown in Fig. 18. A very complex interaction between the shock-wave system and the chemically reacting boundary layer is observed. Complete combustion is achieved behind the shock being reflected from the projectile surface as shown in Fig. 19, which presents water vapor mass fraction contours. At a higher Mach number, $M=8$, combustion takes place along the entire boundary layer in the nose region of the projectile, as shown in Fig. 20. Complete combustion is achieved in this case behind the first reflected shock wave from the tube wall, as shown in Fig. 21 which shows water vapor mass fraction contours. The low density reacting boundary layer existing in the $M=7.2$ and $M=8$ cases tends to separate much easier than a nonreacting boundary layer when a shock wave impinges upon it, as was previously explained. Figure 22 shows the variation of skin friction coefficient along the projectile surface for these two flows. In both cases, the boundary layer separates when the first reflected shock wave impinges on the projectile surface. The separation bubble for the $M=8$ case is much larger due to a stronger impinging shock wave. For the $M=7.2$ case there is a second separation at the location where the second reflected shock wave, strengthened by the combustion process being completed, impinges on the projectile surface.

Finally, a study was conducted on the effects of injecting nitrogen into the boundary layer in an attempt to prevent premature combustion in the nose region. The calculation was carried out for the $M=7.2$ case. The nitrogen was injected at a temperature of $600^{\circ} \mathrm{K}$ along the projectile nose at a uniform nondimensional mass flow rate $F=(\rho v)_{w} /(\rho u)_{\infty}=2.2 \times 10^{-2}$. Figure 23a shows water vapor mass fraction contours and should be compared with the previous result shown in Fig. 19 for zero gas injection. Figure 23b shows nitrogen mass fraction contours indicating the penetration distance of the injected gas. The results show that, under the present conditions, the injection of nitrogen had a negative effect, resulting in increased combustion in the boundary layer. A closer look at the boundary layer profiles with and without injection (Fig. 24a) shows that the effect of nitrogen injection was merely to shift the combustion region away from the surface. This resulted in a closer coupling between the shock and combustion zone, which forced a slight rotation of the shock wave. This in turn enhanced even more the combustion process along the boundary layer, producing higher temperatures (Fig. 24b). It is possible that with significantly higher injection rates, combustion along the nose boundary layer could eventually be quenched, however, this was not determined in the present study.

\section{Application of Vector Extrapolation Methods}

The test case consisting of a supersonic flow over a compression corner for a stoichiometric mixture of hydrogenair discussed previously was also computed using RRE and MPE for a Mach number $M=4$. The combined code (iterative scheme and extrapolation method) was first run without chemical reactions. Figure 25a shows the density residual history obtained by applying RRE in the cycling mode with $K M A X=20$ after 80 and 200 initial iterations. It can be seen that better results are obtained for this problem by employing RRE early on. This seems to be true for nonlinear problems in general. Figure $25 \mathrm{~b}$ contains the residual history obtained by using RRE again in the cycling mode with $K M A X=10,20,30$ after 80 initial iterations. Note that the convergence rates obtained with these three values of $K M A X$ are very similar, and therefore, the smallest value of $K M A X$ is selected for subsequent calculations since it requires the minimum amount of storage.

The code was next run with chemical reactions. Figure 26 gives the convergence history obtained using RRE and MPE in the cycling mode with $K M A X=10$ and after 200 initial iterations. It can be seen that RRE converges slightly faster than MPE. A reduction of approximately 5 orders of magnitude is achieved without extrapolation in 
1000 iterations, whereas with RRE and MPE this takes only 620 and 670 iterations respectively. The overhead in CPU time due to the use of extrapolation turned out to be very small (less than 1\%) in all the above cases, with approximately $30 \%$ increase in storage requirements for the $K M A X=10$ case.

\section{Conclusions}

A new computational fluid dynamics code has been developed for solving the fully coupled twodimensional/axisymmetric Reynolds-averaged NavierStokes equations and species continuity equations in an efficient manner. It employs the LU-SSOR implicit factorization scheme and a second-order symmetric TVD differencing scheme. Results show that this characteristicbased scheme improves convergence and shock resolution relative to the more classical centered-differenced schemes with artificial dissipation. Vector extrapolation methods used in combination with the basic iterative scheme significantly improved the convergence rate and resulted in a very small overhead in CPU time. The code has been used to study shock wave/boundary layer interactions in a ram accelerator configuration. Results indicate a new combustion mechanism in which a shock wave induces combustion in the boundary layer, which then propagates outwards and downstream. At higher Mach numbers, spontaneous ignition in part of the boundary layer was observed, which eventually extended along the entire boundary layer at still higher values of the Mach number.

The present results suggest that viscous effects can strongly affect the performance of the various hypersonic propulsion systems presently under consideration. A more systematic analysis should be conducted as a continuation of this study. Other important topics, such as better turbulence modeling and proper treatment of the turbulence-chemistry interactions, are challenging areas that require further exploration.

\section{Acknowledgments}

The author would like to thank Dr. Jian-Shun Shuen of the NASA Lewis Research Center for his help during the development of the computer code. Thanks are also due to Dr. Avram Sidi of the Technion-Israel Institute of Technology for his guidance during the implementation of his vector extrapolation code. Support to this work was provided by the Numerical Aerodynamic Simulation Program (NAS).

\section{References}

1. Adamson, T.C. and Messiter, A.F., "Analysis of TwoDimensional Interactions Between Shock Waves and Boundary Layers," Annual Review of Fluid Mechanics, Vol. 12, 1980, pp. 103-138.

2. Delery, J. and Marvin, J.G., "Shock-Wave Boundary Layer Interactions," AGARD-AG-280, February 1986.

3. Hamed, A. and Shang, J.S., "Survey and Assessment of Validation Data Base for Shockwave Boundary Layer Interactions in Supersonic Inlets," AIAA Paper 89-2939, July 1989.

4. Ferri, A. and Agnone, A.M., "Heat Conduction Controlled Combustion for Scramjet Applications," AIAA Paper NYU-AA-73-18.

5. Ostrander, M.J., Hyde, J.C., Young, M.F., Kissinger, R.D., and Pratt, D.T., "Standing Oblique Detonation Wave Engine Performance," AIAA Paper 87-2002, June 1987.

6. Townend, L.H., "Detonation Ramjets for Hypersonic Aircraft," Royal Aircraft Establishment, Farnborough, England, TR 70218, November 1970.

7. Morrison, R.B., "Oblique Detonation Wave Ramjet Report," Universal Systems Inc., Contract NAS114771, January 1978.

8. Menees, G.P., Adelman, H.G. and Cambier, J.L., "Analytical and Experimental Investigations of the Oblique Detonation Wave Engine Concept," Agard PEP 75th Symposium on Hypersonic Combined Cycle Propulsion, Madrid, Spain, May 1990.

9. Hertzberg, A., Bruckner, A.P. and Bogdanoff, D.W., "Ram Accelerator : A New Chemical Method for Accelerating Projectiles to Ultrahigh Velocities," AIAA Journal, vol. 26, Feb. 1988, pp 195-203.

10. Bruckner, A.P., Bogdanoff, D.W., Knowlen, C. and Hertzberg, A., "Investigations of Gasdynamic Phenomena Associated with the Ram Accelerator Concept," AIAA Paper 87-1327, June 1987.

11. Knowlen, C., Bruckner, A.P., Bogdanoff, D.W. and Hertzberg, A., "Performance Capabilities of the Ram Accelerator," AIAA Paper 87-2152, June 1987.

12. Bruckner, A.P., Knowlen, C., Hertzberg, A. and Bogdanoff, D.W., "Operational Characteristics of the Thermally Choked Ram Accelerator," to be published in Journal of Propulsion and Power. 
13. Yungster, S., Eberhardt, S. and Bruckner, A.P., "Numerical Simulation of Shock-Induced Combustion Generated by High Speed Projectiles in Detonable Gas Mixtures," AIAA Paper 89-0673, 1989.

14. Yungster, S. and Bruckner, A.P., "A Numerical Study of the Ram Accelerator Concept in the Superdetonative Velocity Range," AIAA Paper 892677, July 1989.

15. Yungster, S., Eberhardt, S. and Bruckner, A.P., "Numerical Simulation of Hypervelocity Projectiles in Detonable Gases," to be published in AIAA Journal.

16. Kull, A., Burnham, E., Knowlen, C., Hertzberg, A. and Bruckner, A.P., "Experimental Studies of Superdetonative Ram Accelerator Modes," AIAA Paper 89-2632, July 1989.

17. Bruckner, A.P., Hertzberg, A., Kull, A.E., Burnham, E.A., Knowlen, C. and Yungster, S., "High Speed Modes of the Ram Accelerator," 40th Meeting of the Aeroballistic Range Association, Paris, France, September 1989.

18. Bruckner, A.P. and Hertzberg, A., "Ram Accelerator Direct Launch System for Space Cargo," Paper No. IAF-87-211, 38th Congress of the International Astronautical Federation, Brighton, England, Oct. 1987.

19. Kaloupis, P. and Bruckner, A.P., "The Ram Accelerator: A Chemically Driven Mass Launcher," AIAA Paper 88-2968, July 1988.

20. Humphrey, J.W., "Parametric Study of an ODW Scramaccelerator for Hypersonic Test Facilities," AIAA Paper 90-2470, July 1990.

21. McBride, B.J. and Shuen, J.S., Private communication, NASA Lewis Research Center, Cleveland, $\mathrm{OH}$, January 1990.

22. Reid, R.C., Prausnitz, J.M. and Sherwood, T.K., "The Properties of Gases and Liquids," 3rd Ed., McGraw Hill, New York 1977.

23. Yungster, S., "Numerical Simulation of ShockInduced Combustion for Application to the Ram Accelerator Concept," Ph.D. Dissertation, Dept. of Aeronautics and Astronautics, University of Washington, October 1989.

24. Sekar, B., Mukunda, H.S. and Carpenter, M.H., "The Direct Simulation of High-Speed Mixing-Layers Without and With Chemical Heat Release," Computational Fluid Dynamics Symposium on Aeropropulsion, NASA CP-10045, Cleveland, OH, April 1990.
25. Baldwin, B. and Lomax, H., "Thin Layer Approximation and Algebraic Model for Separated Turbulent Flows," AIAA Paper 78-257, January 1978

26. Cambier, J.L., Adelman, H. and Menees, G.P., "Numerical Simulations of an Oblique Detonation Wave Engine," AIAA Paper 88-0063, Jan. 1988.

27. Jameson, A. and Turkel, E., "Implicit Schemes and LU Decompositions," Math. Comp., 37, 1981, pp. 385-397.

28. Yoon, S. and Jameson, A., "An LU-SSOR Scheme for the Euler and Navier-Stokes Equations," AIAA Paper 87-600, Jan. 1987.

29. Jameson, A. and Yoon, S., "Lower-Upper Implicit Schemes with Multiple Grids for the Euler Equations," AIAA Journal, Vol. 25, July 1987, pp. 929935 .

30. Shuen, J.S. and Yoon, S., "Numerical Study of Chemically Reacting Flows Using a Lower-Upper Symmetric Successive Overrelaxation Scheme," AIAA Journal, Vol. 27, December 1989, pp. 17521760 .

31. Harten, A., "On a Class of High Resolution TotalVariation-Stable Finite Difference Schemes," SIAM J. Num. Anal., Vol. 21, 1984, pp. 1-23.

32. Yee, H.C., "Upwind and Symmetric ShockCapturing Schemes," NASA TM-89464, May 1987.

33. Park, C. and Yoon, S., "A Fully-Coupled Implicit Method for Thermo-Chemical Nonequilibrium Air at Sub-Orbital Flight Speeds," AIAA Paper 89-1974CP, 1989.

34. Tsai, P.Y. and Hsieh, K.C., "Comparative Study of Computational Efficiency of Two LU Schemes for Non-Equilibrium Reacting Flows," AIAA Paper 900396, January 1990.

35. Yee, H.C., Klopfer, G.H. and Montagnè, J.-L., "High-Resolution Shock-Capturing Schemes for Inviscid and Viscous Hypersonic Flows," NASA TM100097, April 1988.

36. Sidi, A. and Celestina, M.L., "Convergence Acceleration for Vector Sequences and Applications to Computational Fluid Dynamics," NASA TM101327, ICOMP-88-17, Aug. 1988.

37. Sidi, A., "Efficient Implementation of Minimal Polynomial and Reduced Rank Extrapolation Methods," NASA TM-103240, ICOMP-90-20, Aug. 1990. 
38. Skebe, S.A., Greber, I. and Hingst, W.R., "Investigation of Two-Dimensional Shock-Wave/BoundaryLayer Interactions," AIAA Journal, Vol. 25, June 1987, pp. 777-783.

39. Reda, D.C. and Murphy, J.D., "Shock WaveTurbulent Boundary Layer Interactions in Rectangular Channels, Part II: The Influence of Sidewall Boundary Layers on Incipient Separation and Scale of the Interaction," AIAA Paper 73-16959, Jan. 1973.

40. Tennekes, H. and Lumley, J.L., "A First Course in Turbulence," MIT Press, Cambridge, 1972, pp. 182186. 


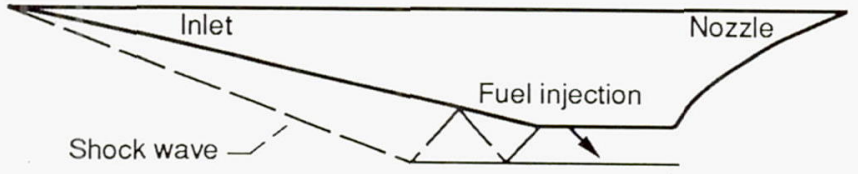

(a) Scramjet engine

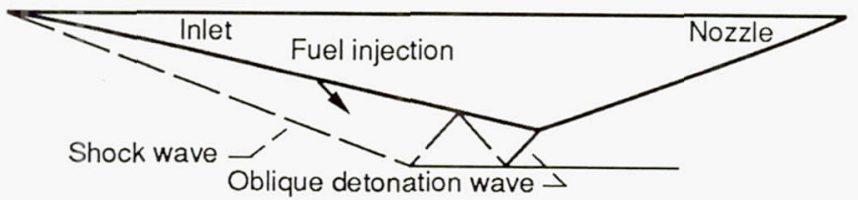

(b) Oblique detonation wave engine.

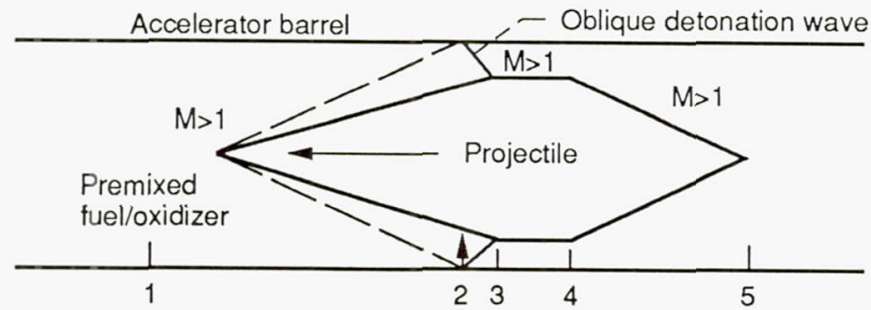

(c) Ram accelerator.

Figure 1.-Hypersonic propulsion schemes.

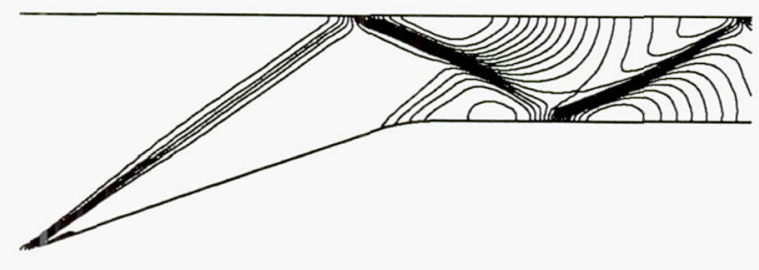

(a) Present method

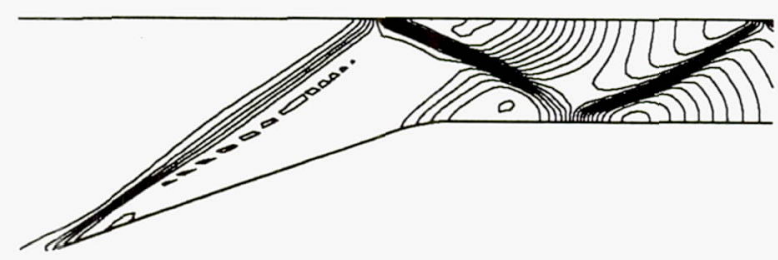

(b) RPLUS

Figure 2.- Pressure contours for an $\mathrm{M}=5$ nonreacting viscous flow past a wedge configuration.

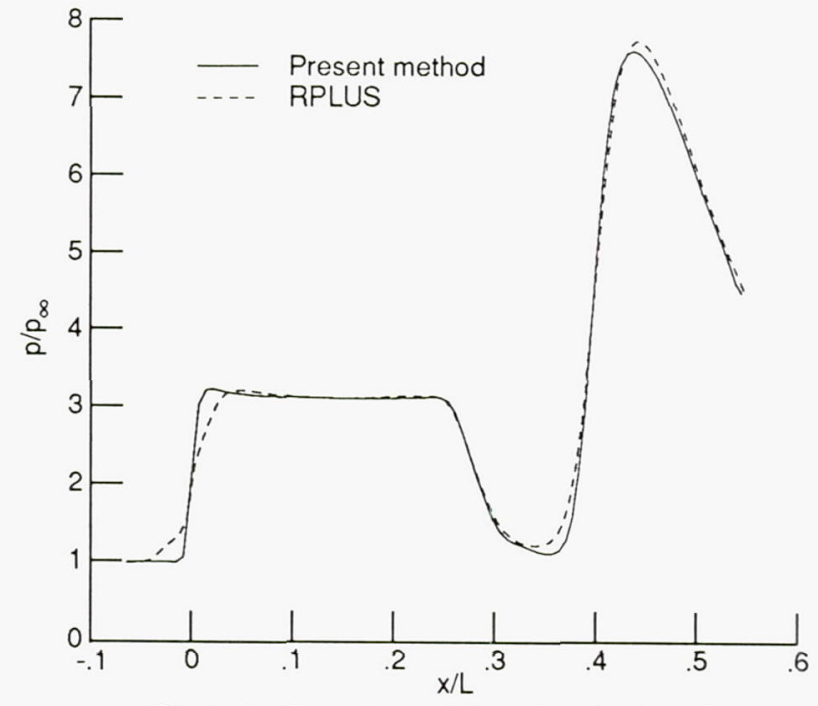

Figure 3.- Pressure variation along body surface.

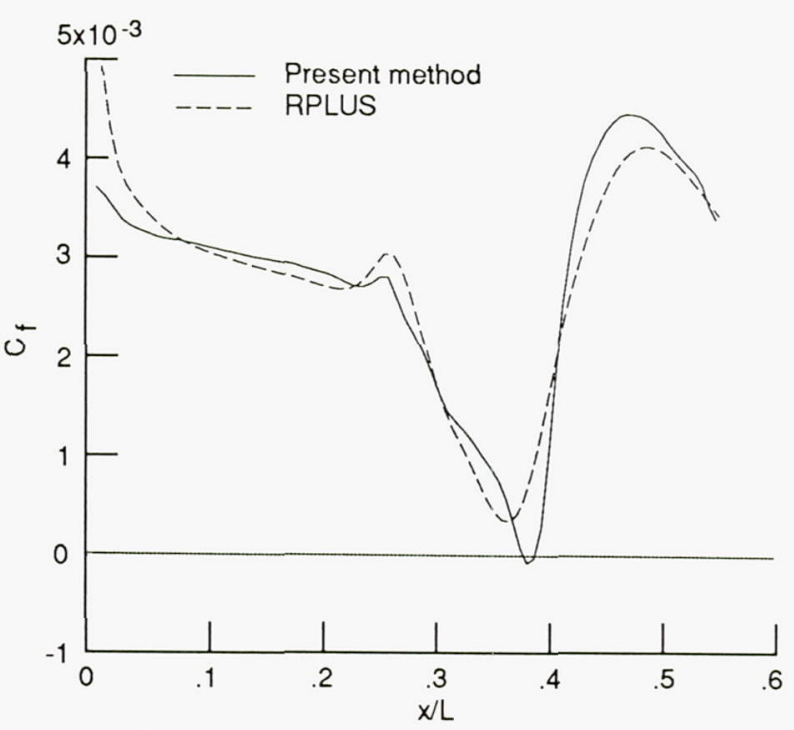

Figure 4.- Skin friction coefficient along body surface. 


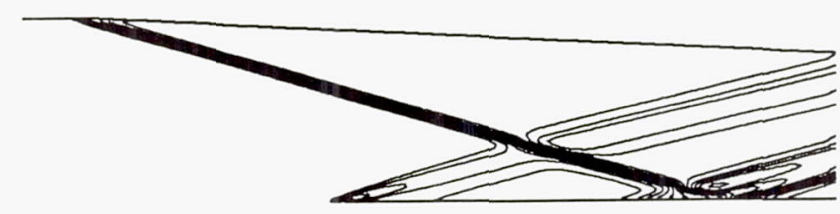

Figure 5.- Pressure contours for laminar shock wave-boundary layer interaction.

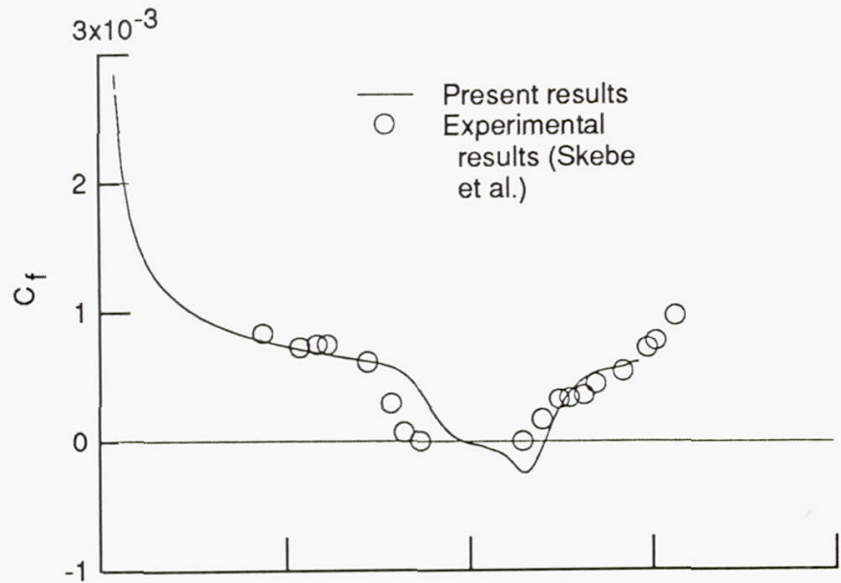

(a) Skin friction coefficient.

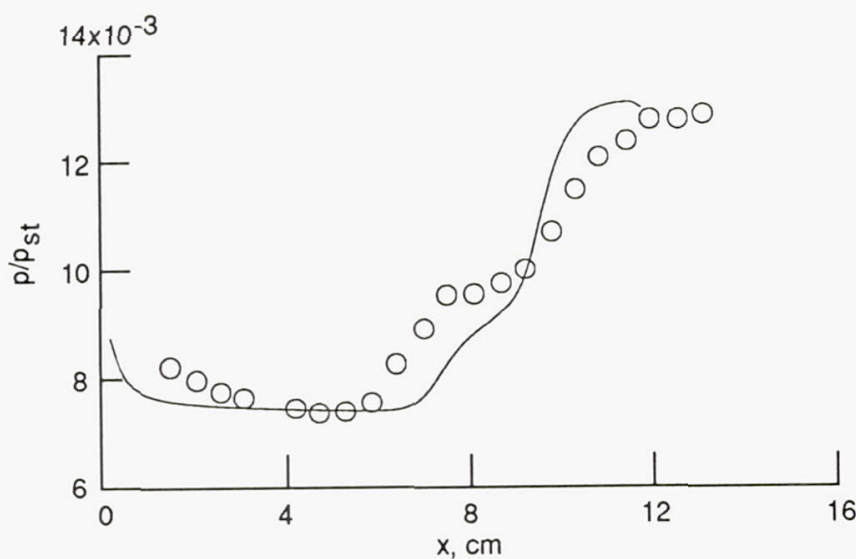

(b) Ratio of static to free-stream total pressure.

Figure 6.- Skin friction coefficient and ratio of static to freestream total pressure along flat plate for laminar flow.

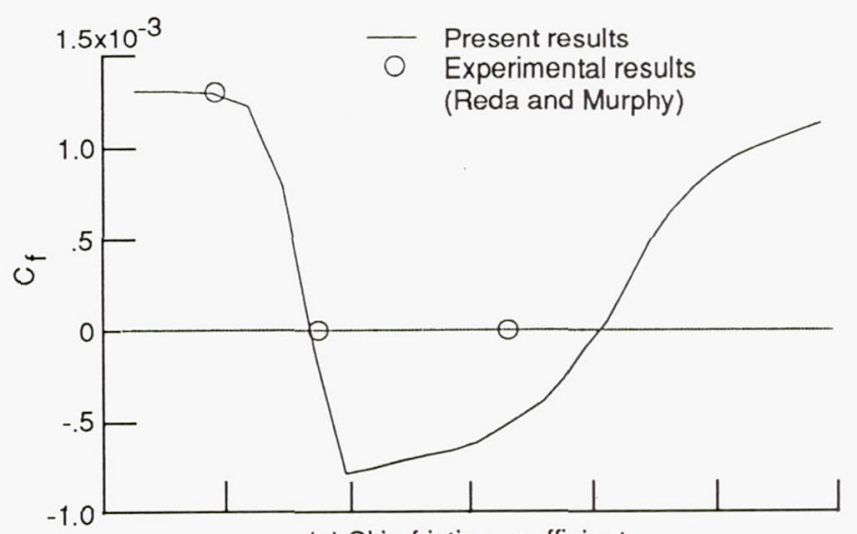

(a) Skin friction coefficient.

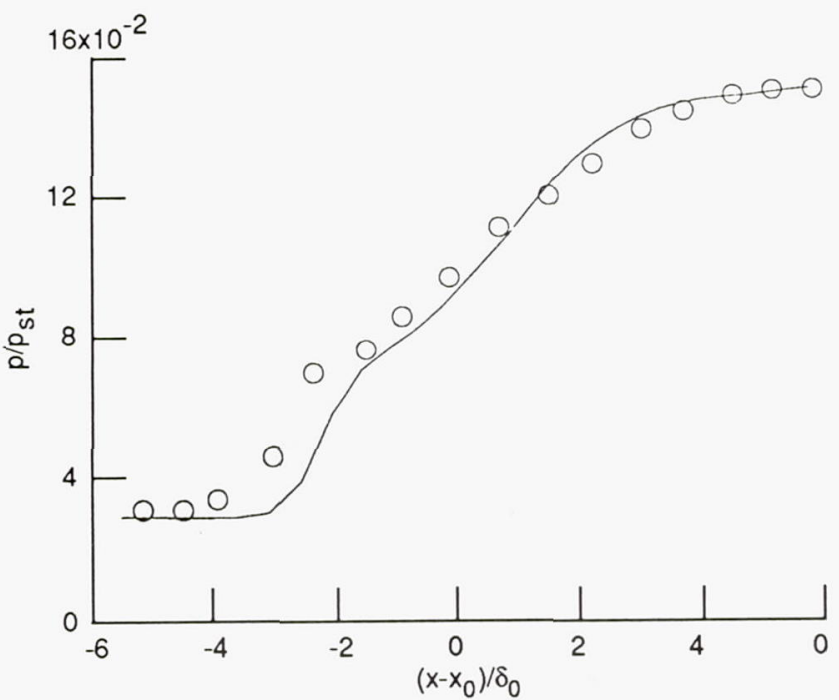

(b) Ratio of static to free-stream total pressure.

Figure 7.- Skin friction coefficient and ratio of static to freestream total pressure along flat plate for turbulent flow. 


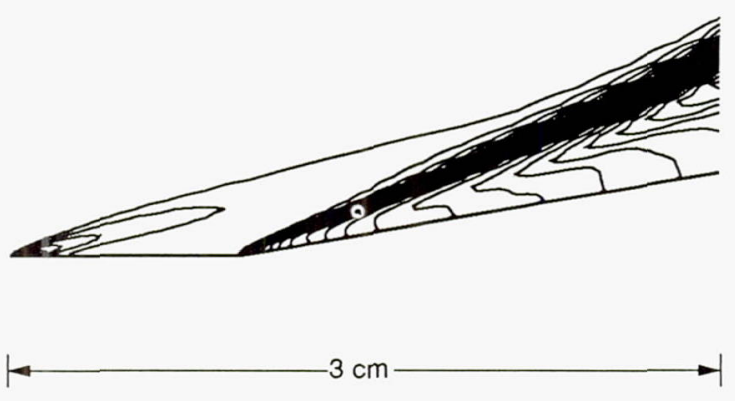

Figure 8.- Pressure contours for an $\mathrm{M}=4.5$ reacting flow past a compression corner $\left(\mathrm{H}_{2}\right.$ /air; $\varphi=1 ; \mathrm{T}_{\infty}=900 \mathrm{~K}$; $\left.P_{\infty}=1 \mathrm{~atm}\right)$

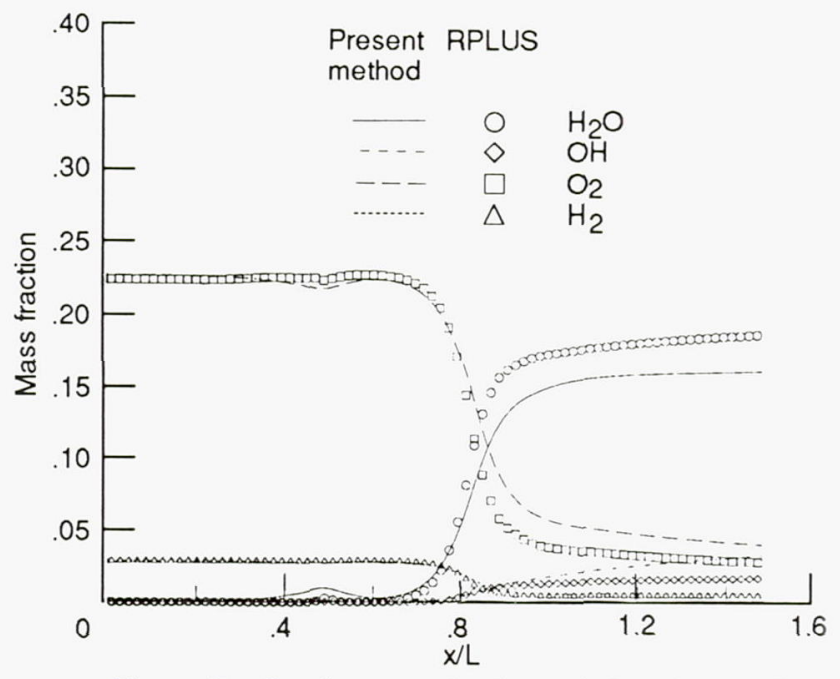

Figure 10.- Species mass fraction variation along gridline located $0.2 \mathrm{~cm}$ from base of ramp.

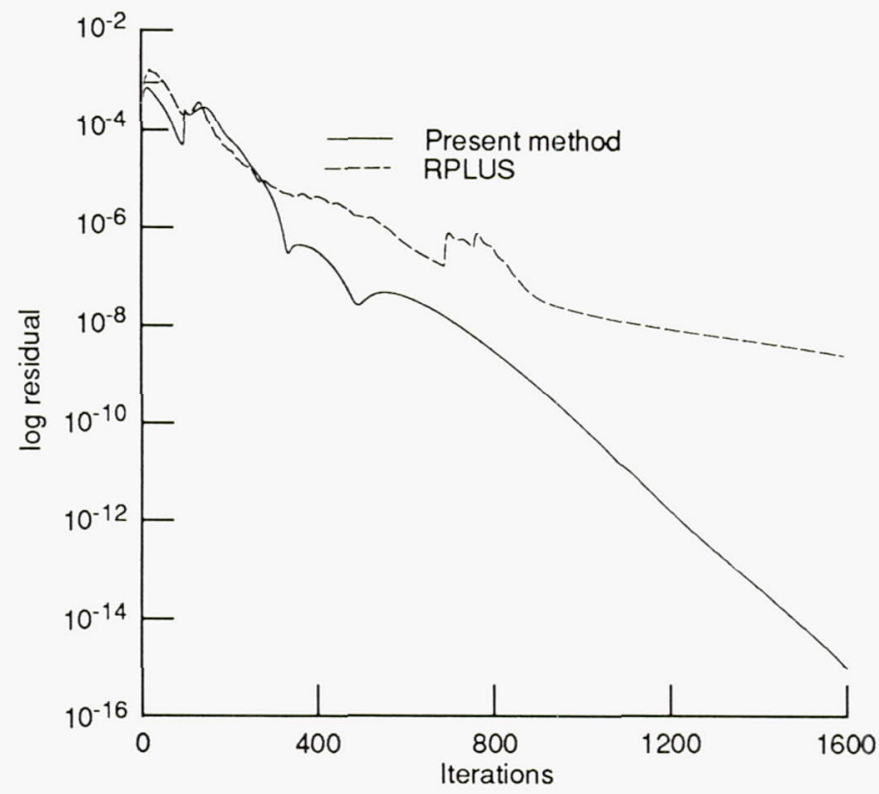

Figure 11.- Convergence history of $L_{1}$ density residual (grid $80 \times 50)$.
Figure 9.- Pressure and temperature variation along gridline located $0.2 \mathrm{~cm}$ from base of $\operatorname{ramp}\left(M=4.5 ; \mathrm{T}_{\infty}=900 \mathrm{~K}\right)$.

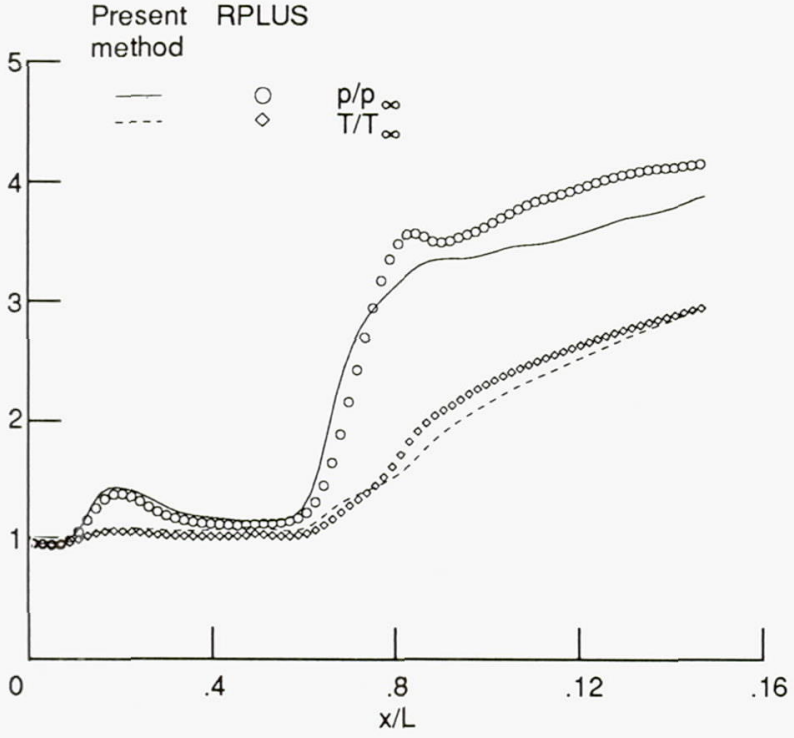




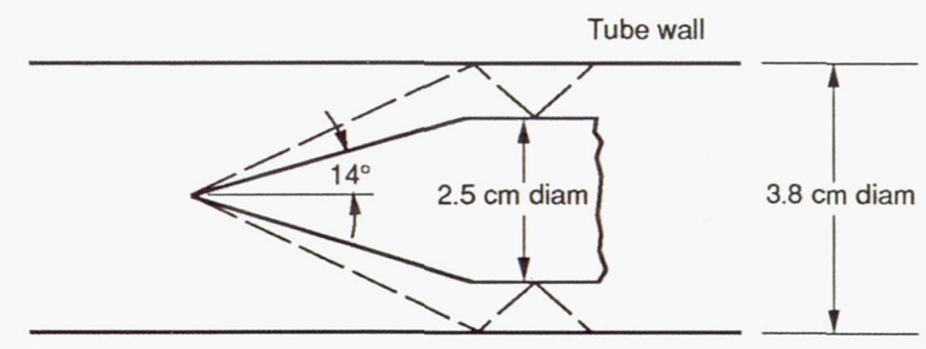

Figure 12.- Ram accelerator configuration used for studying shock wave/ boundary layer interactions $\left(\mathrm{H}_{2} / \mathrm{air} ; \mathrm{T}_{\infty}=300 \mathrm{~K} ; \mathrm{P}_{\infty}=1 \mathrm{~atm}\right)$.

\section{CONTOUR LEUELS}

1. 15000

1. 30000

1.45000

1. 60000

1. 75000

1. $900 \mathrm{0}$

2. 05000

2. 20000

2. 35000

2.50000

2.65000

80000

3.10000

3.25000

3. 40000

3.55000

3. 7 वण00

3. 85 เ60

4. ㄸำ0

4. 150 무

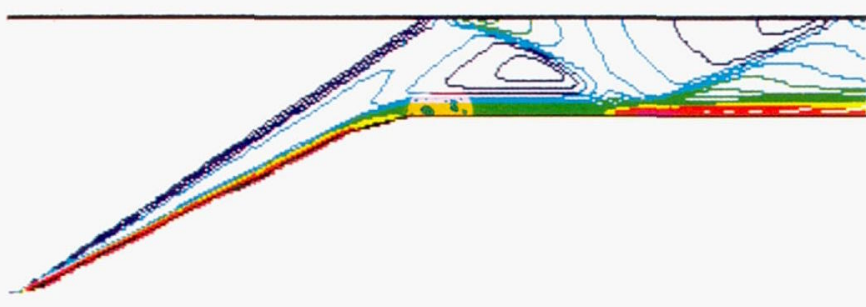

Figure 13.- Nondimensional temperature contours $\left(T / T_{\infty}\right)$ for nonreact ing $M=6.7$ flow (for clarity, vertical direction is magnified by a factor of 2).

\section{CUNTOUR LEUELS}

1.30000

1. 60000

1. 90000

2. 20000

2. 50000

2. 8पणवप

3. 1 पवण

3. 4 पणतण

3. 7वपण0

4. पवपवर

4. 30000

4. 60000

4. 9घாत0

5. 20000

5.50000

5.80000

6. 10000

6. 40000

?. 00000

7. 90000

8.20000

8. 50000

8. 80000

9. 1 पवण

9. 4 घणघए

9. 7पणतण

1व. पवपण

10. 3ดปด

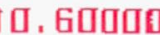

เั. 9ยवप⿴囗十

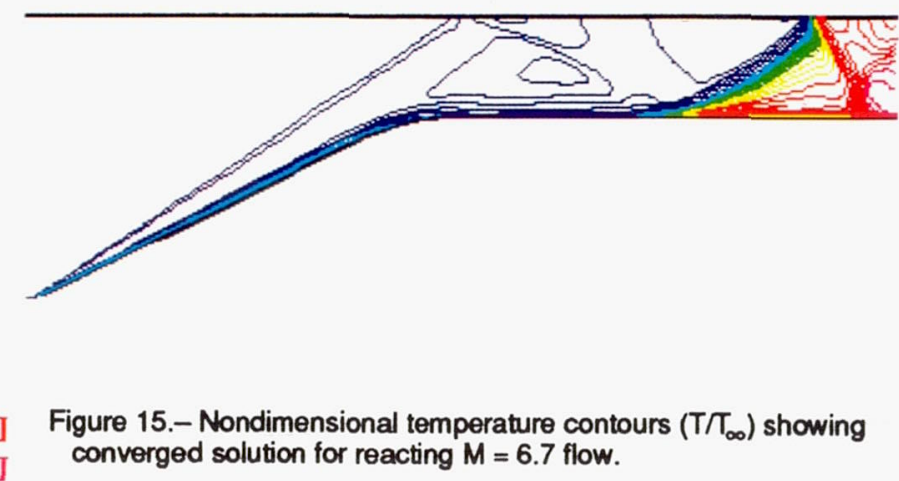

Figure 14.- Nondimensional temperature contours $\left(T / T_{\infty}\right)$ for reacting $M=6.7$ flow after 100 iterations. 


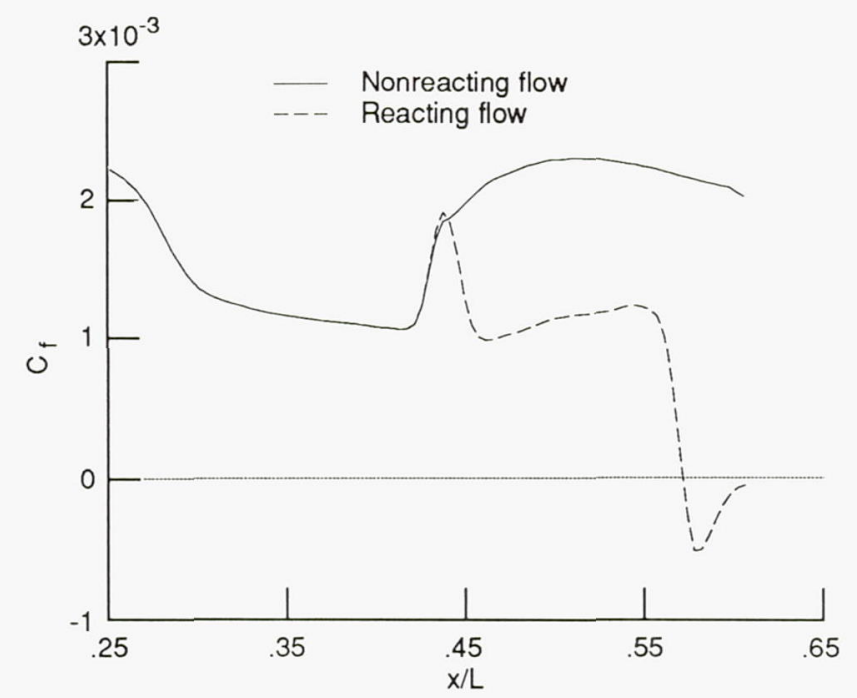

Figure 16.- Skin friction coefficient along projectile surface.

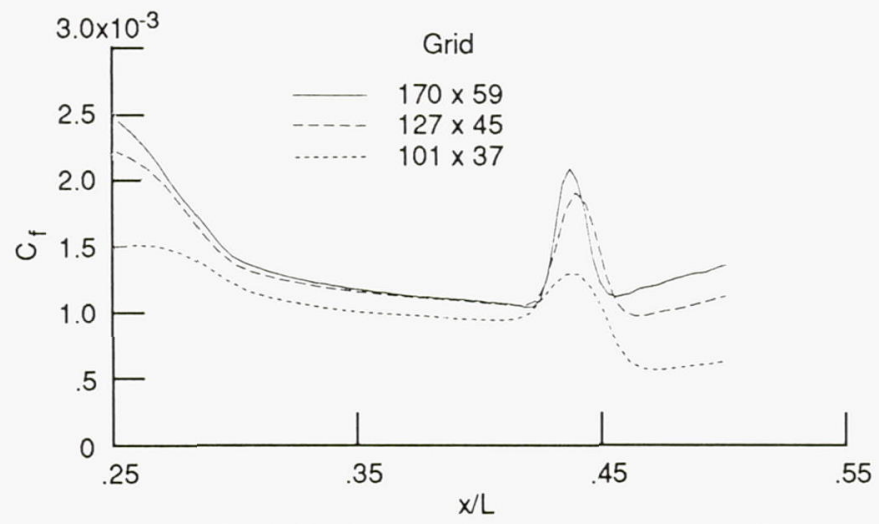

Figure 17.- Grid refinement for ram accelerator configuration. 
CONTOUR LEUELS

D. 01000

อ. 02000

0. 03000

‥ 04000

0. 06000

0. 07000

0. 08000

0. 09000

0. 10000

0. 11000

0. 12000

0. 13000

1. 30000

1. 90000

2. 20000

2. 50000

2. 80000

3. 10000
3. 40000

3.70000

4. 00000

4.30000

4. 60000

4. 90000

5. 200000

5. 80000

6. 10000

6. - 10000

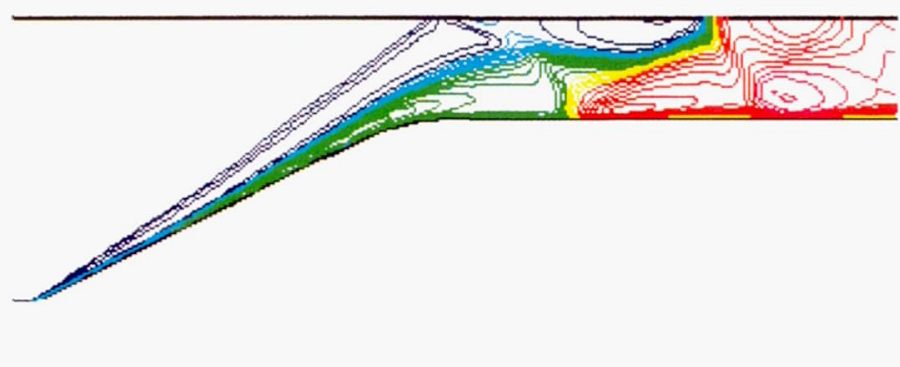

․ 17000

0. 18000

0.20000

0. 21000

๑. 2 2पव口

0.23 पव

0.24000

0. 25000

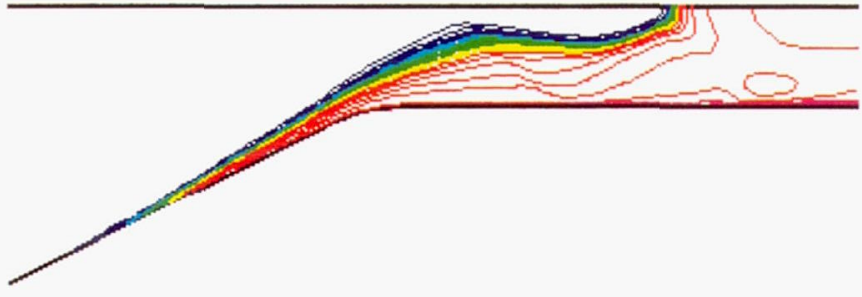

Figure 19.- Water vapor mass fraction contours for reacting $M=7.2$

Figure 18.- Nondimensional temperature contours showing converged solution for reacting $M=7.2$ flow.

7. 90000

8. 20000

8. 50000

8.80000

9. 10000

9. 40000

9. ร000

10. 00000

10. 3000

10 . 60000

10. 90000

CONTOUR LEUELS

1. 40000

1.80000

2. 20000

2. 60000

3. 00000

3. 40000

4. 20000

4. 60000

5. 00000

5. 40000

5. 80000

6. 20000

6. 60000

7. 00000

?. 8000

8. 60000

9. 00000

9. 40000

9. 80000

10. 2000

10. 60000

11. वणवूव

11.80000

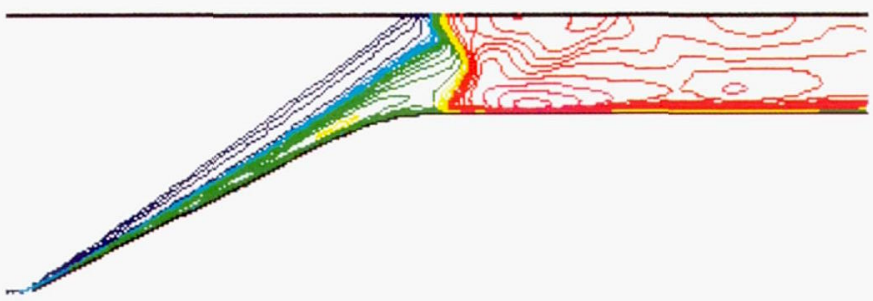

Figure 20 - Nondimensional temperature contours showing converged solution for reacting $\mathrm{M}=8.0$ flow. 
CONTOUR LEUELS

0. 01000

0.02000

0.04000

ด. 05000

๑. 06000

0. 08000

D. 09000

0. 10000

0. 12000

定: 13000

(1.) 15000

0. 17000

D. 18000

0. 19000

0.20000

0.21000

0. 22 पव

0. 2300

0. 24000

0. $2500 \square$

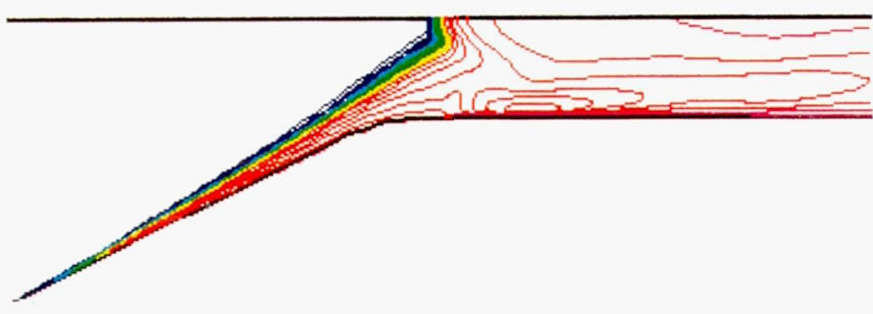

Figure 21. - Water vapor mass fraction contours for reacting $M=8.0$

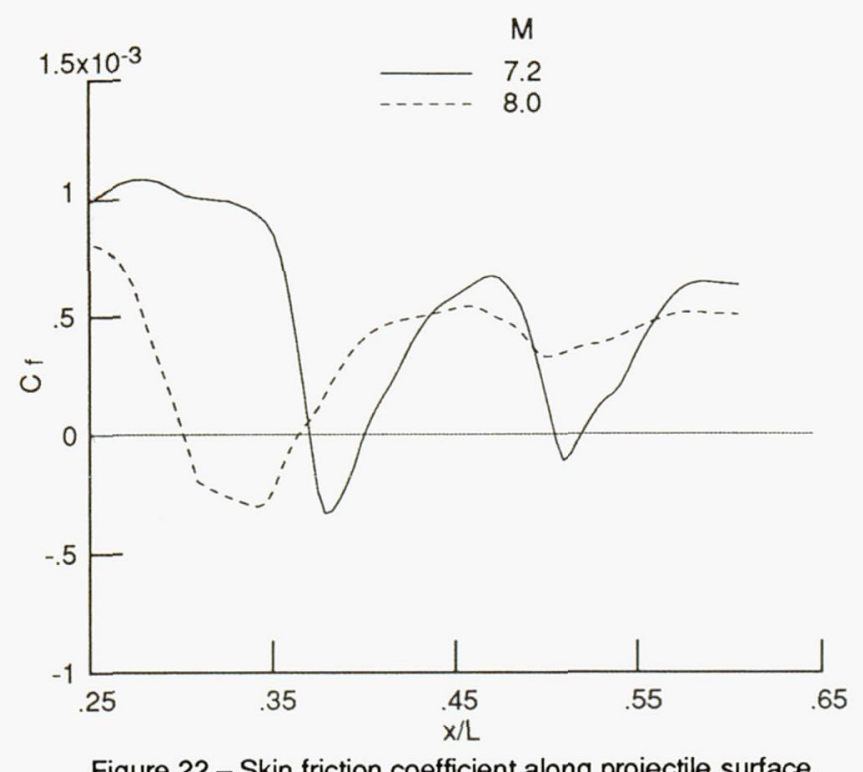

CONTOUR LEUELS

D. 01000

0. 03000

0. 04000

0. 05000

0. 06000

0. $0>000$

0. 08000

0. 09000

11000

0. 12000

0. 16000

0.17000

D. 18000

0. 19000

0. 20000

․ 21 पᄆ口

0.23000

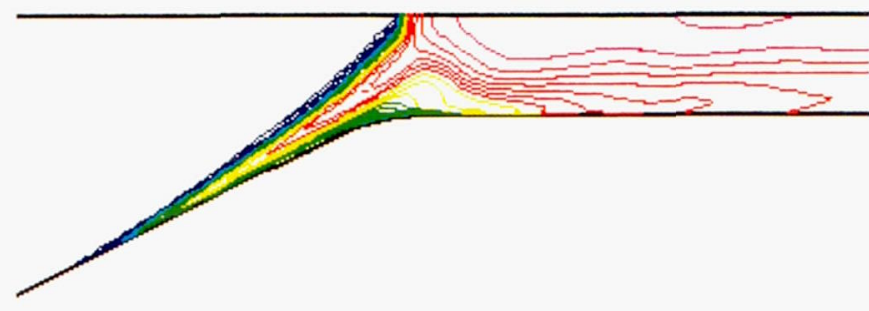

(a) Water vapor mass fraction.

\section{CONTOUR LEUELS}

0.75000

0.76000

$0 .>7000$

0. 78000

0. 79000

․ 80000

0. 82000

0. 83000

0.84000

0. 85000

0.87000

0. 88000

0.91000

0.92000

. 94000

0. 95000

0. 9600

0.970ロ

อ. 9800

0. 9900

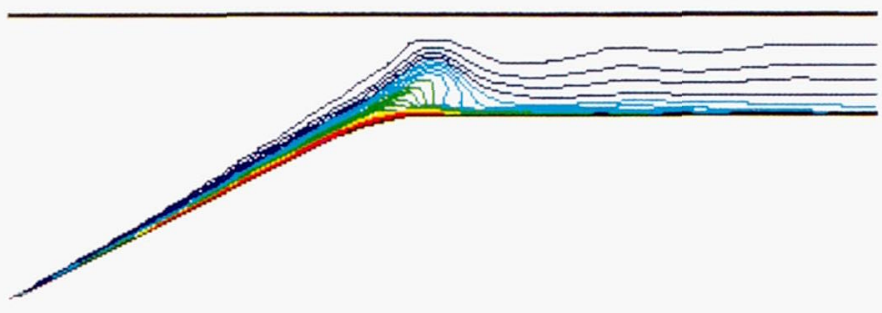

(b) Nitrogen mass fraction.

Figure 23.- Water vapor and nitrogen mass fraction contours for reacting $M=7.2$ flow with mass injection. 


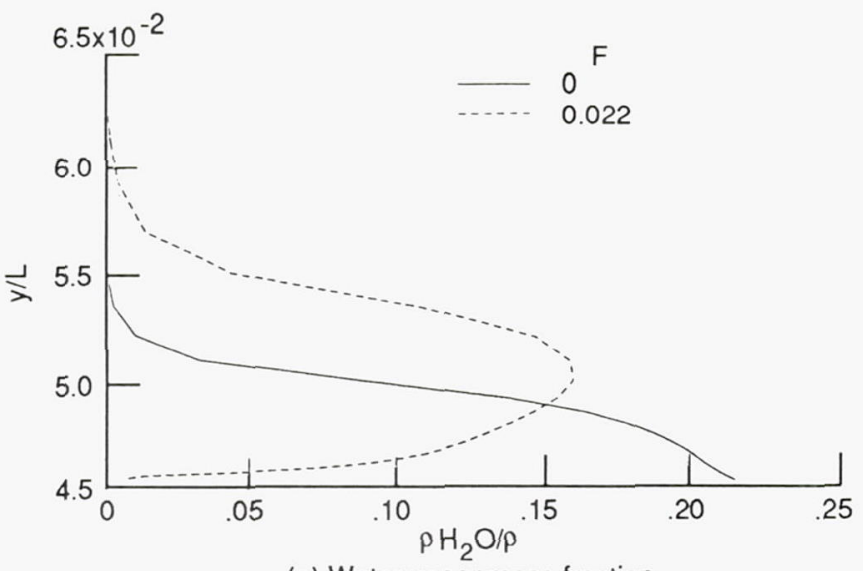

(a) Water vapor mass fraction.

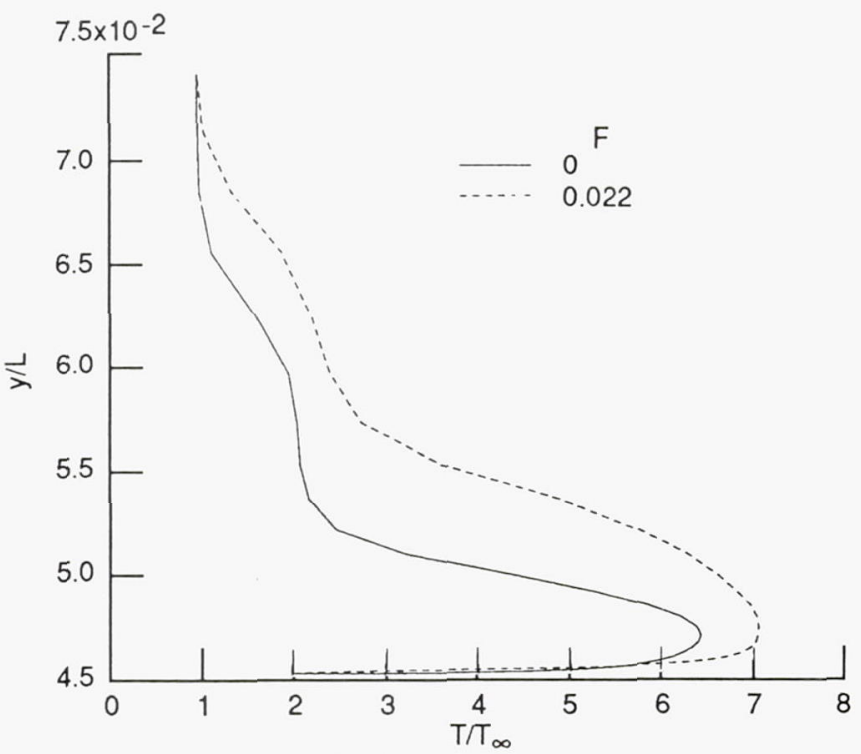

(b) Nondimensional temperature.

Figure 24.- Water vapor mass fraction and nondimensional temperature profiles in boundary layer at station $x / L=0.18$ $\left(F=(\rho v)_{W} /(\rho u)_{\infty}, M=7.2, T_{W}=600 \mathrm{k}\right)$.

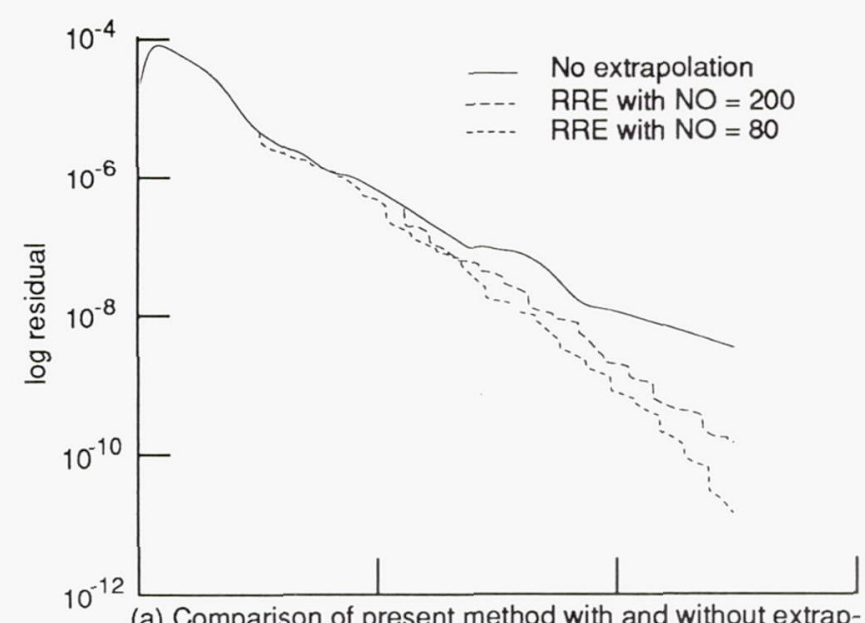

(a) Comparison of present method with and without extrapolation, for $\mathrm{KMAX}=20$.

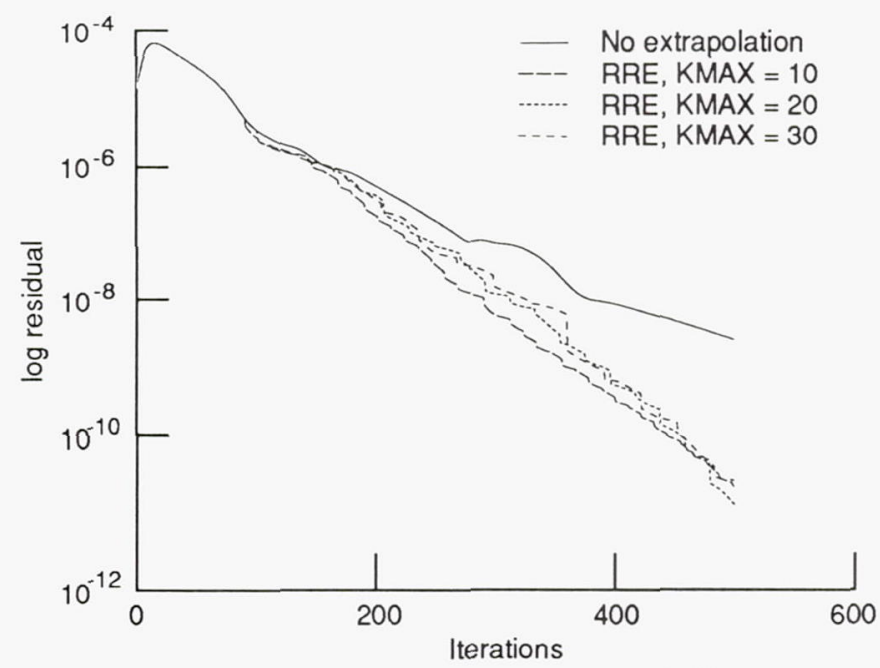

(b) Comparison of present method with and without extrapolation, for $\mathrm{NO}=80$.

Figure 25.- Convergence history of $L_{2}$ density residual for nonreacting flow (grid, $80 \times 50$ ).

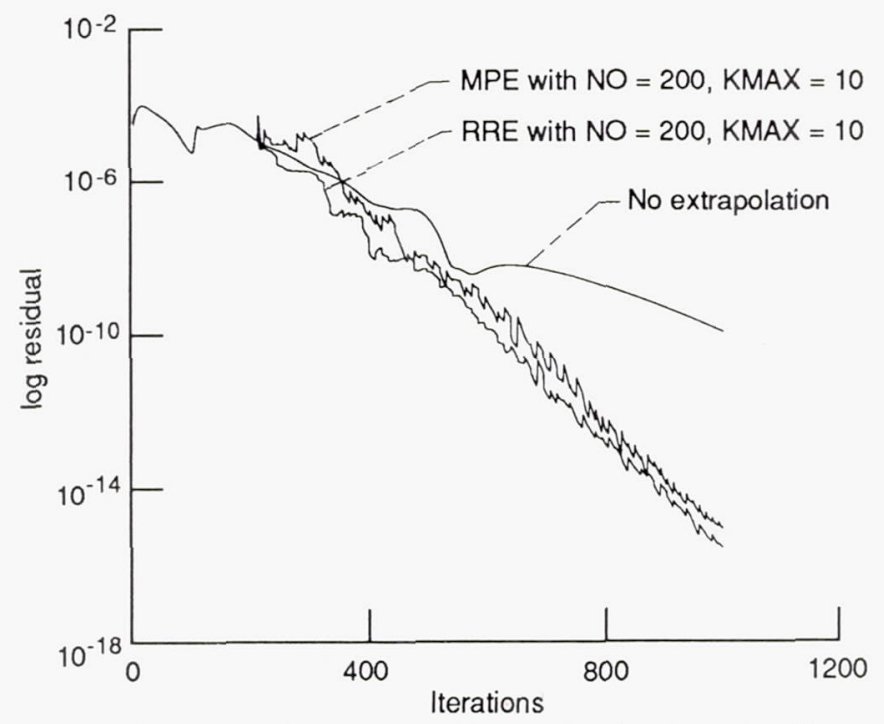

Figure 26.- Convergence history of $L_{2}$ density residual for reacting flow (grid, $80 \times 50$ ). 


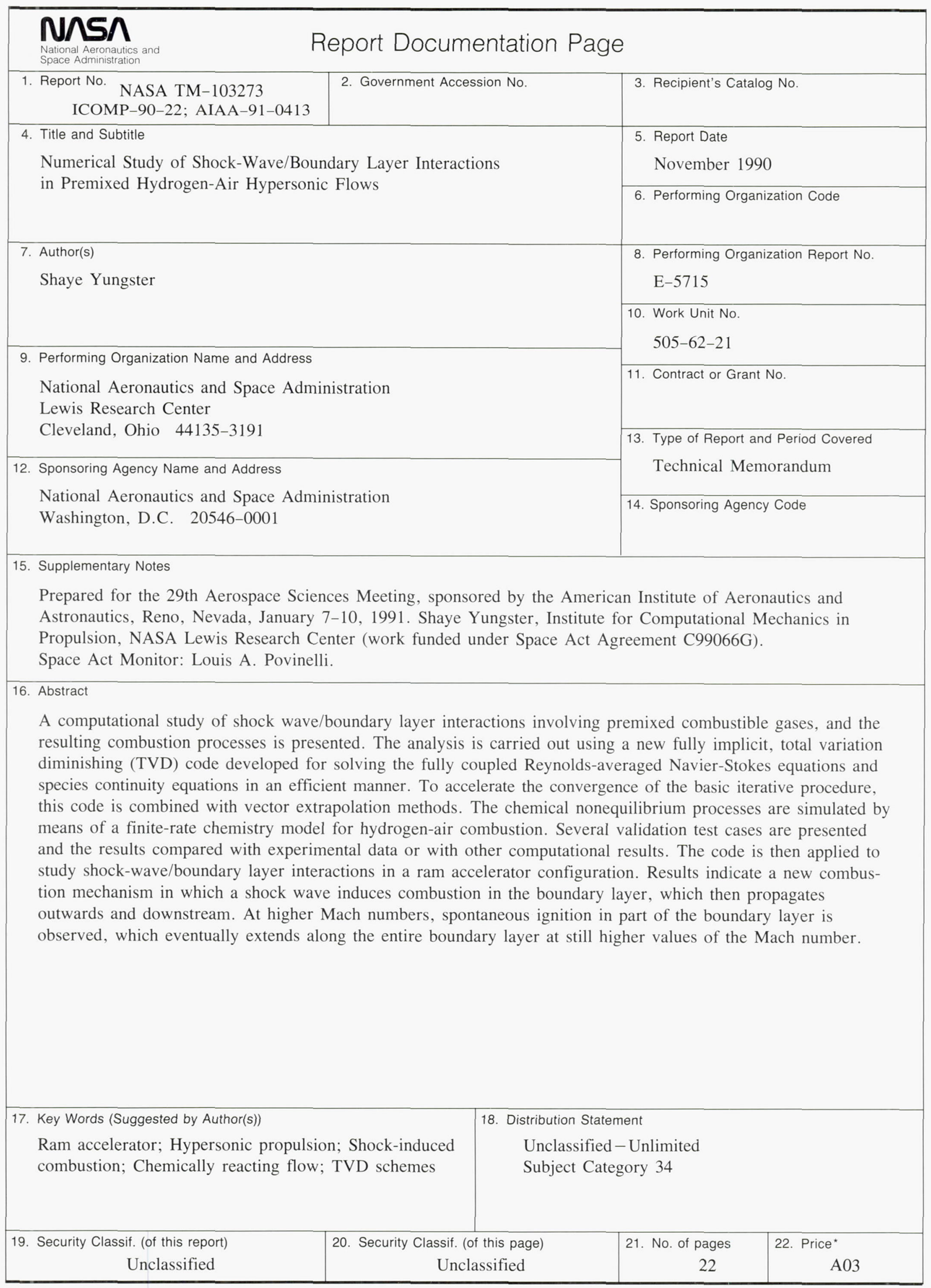

